\title{
Two Novel
}

Diastereoselective

Three-Component

Reactions of Alkenes or

3,4-Dihydro-(2H)-Pyran

with Urea/Thiourea -

Aldehyde Mixtures:

[4+2]-Cycloaddition Vs

Biginelli-Type Reaction

Yulin Zhu, ${ }^{\dagger}$ Shenlin Huang, ${ }^{\dagger}$ Jieping Wan, ${ }^{\dagger}$ Lei Yan, ${ }^{\dagger}$ Yuanjiang Pan, ${ }^{*}{ }^{\dagger}$ and Anxin $\mathrm{Wu}^{\star, \neq}$

${ }^{\dagger}$ Department of Chemistry, Zhejiang University, Hangzhou 310027, P.R.China

${ }^{*}$ Key Laboratory of Pesticide and Chemical Biology of Ministry of Education, Central China Normal University, Wuhan 430079, P.R.China

cheyjpan@zju.edu.cn; chwuax@mail.ccnu.edu.cn

\section{Table Contents}

General Details

Spectral Data

${ }^{1} \mathrm{H}$ and ${ }^{13} \mathrm{C}$ NMR Spectra
S1

S2-8

S9-27

General: All chemicals were obtained from commercial suppliers and used without further purification. Anhydrous conditions are not required for the reaction. Melting points were determined using XT-4 apparatus and were not corrected. ${ }^{1} \mathrm{H}$ and ${ }^{13} \mathrm{C}-\mathrm{NMR}$ spectra were recorded on Bruker AVANCE DMX-500 spectrometry at $500 \mathrm{MHz}$ and $125 \mathrm{MHz}$ in DMSO- $d_{6}$, respectively. Chemical shifts are reported in ppm $(\delta)$, relative to the internal standard of tetramethylsilane (TMS). $1 \mathrm{D}{ }^{1} \mathrm{H}$ and ${ }^{13} \mathrm{C}$ NMR spectra were acquired under standard conditions ( $5 \mathrm{~mm}$ QNP probe). Mass spectra were performed on a Bruker Esquire $3000^{\text {plus }}$ mass spectrometer (Bruker-Franzen Analytik GmbH Breman, Germany) 
equipped with ESI interface and ion trap analyzer. HRMS were obtained on a Bruker 7-tesla FT-ICR MS equipped with an electrospray source (Billelica, MA, USA).

General Procedure: A solution of benzaldehyde 1a (5 mmol), styrene 2 a (5 mmol), and thiourea (6 mmol) in anhydrous $\mathrm{DMF} / \mathrm{CH}_{3} \mathrm{CN}(1.5 \mathrm{~mL} / 6 \mathrm{~mL})$ containing $\mathrm{TMSCl}(5 \mathrm{mmol})$ was refluxed till the reaction was completed. The product $\mathbf{4 a}$ was precipitated directly. Then the crude product was isolated by filtered through a Buechner funnel and was recrystallized from $\mathrm{C}_{2} \mathrm{H}_{5} \mathrm{OH}$ to give pure product.

\section{Spectroscopic data for all compounds:}
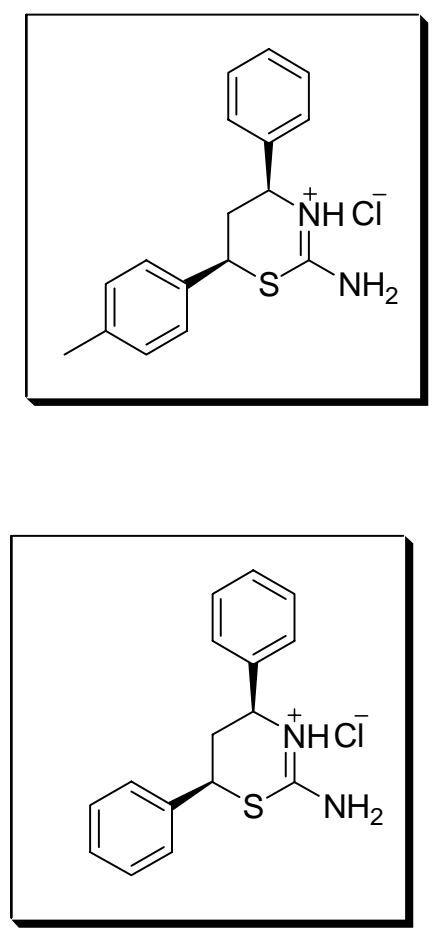

4a: m.p. $295-298^{\circ} \mathrm{C} .{ }^{1} \mathbf{H}$ NMR (500 MHz, DMSO- $\left.d_{6}\right) \delta 10.60$ (s, 1H), 9.58(s, 1H), 9.04(s, 1H), 7.21-7.49(m,9H), 5.07(t, 1H), 4.95(t, $1 \mathrm{H}), 2.50(\mathrm{~m}, 2 \mathrm{H}), 2.30(\mathrm{~s}, 3 \mathrm{H}) ;{ }^{13} \mathrm{C}$ NMR $(125 \mathrm{MHz}$, DMSO-d6) $\delta 167.1,139.5,138.4,134.3,129.6,128.8,128.4$, 127.8, 126.8, 56.8, 44.3, 37.4, 20.7. IR (KBr): 3423, 3026, 1637, 1598, $1481 \mathrm{~cm}^{-1}$. MS (ESI) m/z283 $\left([\mathrm{M}+\mathrm{H}]^{+}\right)$.

4b: m.p. $279-281{ }^{\circ} \mathrm{C} .{ }^{1} \mathbf{H}$ NMR $\left(500 \mathrm{MHz}\right.$, DMSO- $\left.d_{6}\right) \delta 10.50$ (s, 1H), $9.52(\mathrm{~s}, 1 \mathrm{H}), 8.80(\mathrm{~s}, 1 \mathrm{H}), 7.39-7.53(\mathrm{~m}, 10 \mathrm{H}), 5.12(\mathrm{t}, 1 \mathrm{H})$, $4.97(\mathrm{t}, 1 \mathrm{H}), 2.50(\mathrm{~m}, 2 \mathrm{H}) ;{ }^{13} \mathbf{C}$ NMR (125 MHz, DMSO- $\left.d 6\right) \delta$ $168.0,140.4,138.3,130.1,129.9,129.8,129.5,128.9,128.0$, 57.8, 45.5, 38.2. IR (KBr): 3432, 3027, 1639, 1596, $1478 \mathrm{~cm}^{-1}$. MS (ESI) m/z $269\left([\mathrm{M}+\mathrm{H}]^{+}\right)$. 

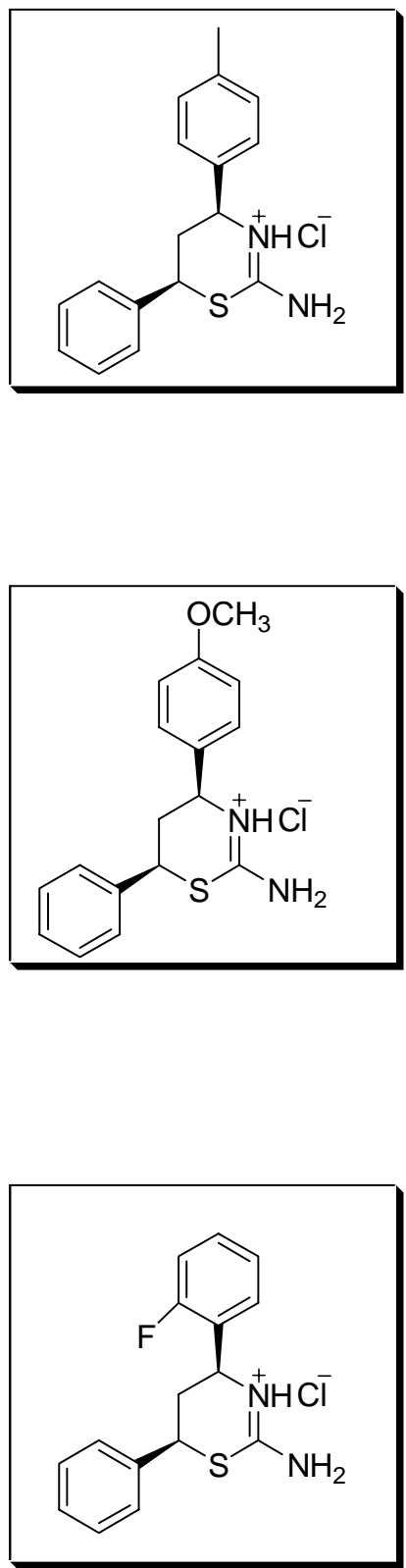

4c: m.p. $276-278{ }^{\circ}$ C. ${ }^{1} \mathbf{H}$ NMR $\left(500 \mathrm{Mz}, \mathrm{DMSO}-d_{6}\right) \delta 10.6(\mathrm{~s}$, 1H), 9.65 (s, 1H), $9.12(\mathrm{~s}, 1 \mathrm{H}), 7.23-7.53(\mathrm{~m}, 9 \mathrm{H}), 5.10(\mathrm{~m}, 1 \mathrm{H})$, $4.92(\mathrm{~m}, 1 \mathrm{H}), 2.45(\mathrm{~m}, 2 \mathrm{H}), 2.31(\mathrm{~s}, 3 \mathrm{H}) ;{ }^{13} \mathrm{C}$ NMR (DMSO-d6, $125 \mathrm{Mz}) \delta 167.9,138.7,138.4,137.4,130.3,130.1,129.9,128.9$, 127.8, 57.5, 45.5, 38.3, 21.7. IR (KBr): 3200, 3033, 1632, 1597 , $1517,1486,11455 \mathrm{~cm}^{-1}$. MS (ESI) m/z $283\left([\mathrm{M}+\mathrm{H}]^{+}\right)$.

4d: m.p. $253-256{ }^{\circ} \mathrm{C} .{ }^{1} \mathbf{H}$ NMR (500 MHz, DMSO- $\left.d_{6}\right) \delta 10.50(\mathrm{~s}$, 1H), $9.55(\mathrm{~s}, 1 \mathrm{H}), 9.00(\mathrm{~s}, 1 \mathrm{H}), 7.38-7.53(\mathrm{~m}, 7 \mathrm{H}), 6.99(\mathrm{~d}, 2 \mathrm{H})$, $5.09(\mathrm{~m}, 1 \mathrm{H}), 4.89(\mathrm{~m}, 1 \mathrm{H}), 3.76(\mathrm{~s}, 3 \mathrm{H}), 2.48(\mathrm{~m}, 2 \mathrm{H}) ;{ }^{13} \mathrm{C}$ NMR (125 MHz, DMSO-d6) $\delta$ 167.8, 160.3, 138.4, 132.2, 130.1, $129.9,129.3,128.9,115.1,57.2,56.2,45.6,38.2$. IR $(\mathrm{KBr})$ : $3205,3031,1632,1606,1585,1516,1251 \mathrm{~cm}^{-1}$. MS (ESI) m/z $299\left([\mathrm{M}+\mathrm{H}]^{+}\right)$.

4e: m.p. $265-267{ }^{\circ}$ C. ${ }^{1} \mathbf{H}$ NMR $\left(500 \mathrm{MHz}, \mathrm{DMSO}-d_{6}\right) \delta 10.77$ (s, 1H), 9.67 (s, 1H), $9.04(\mathrm{~s}, 1 \mathrm{H}), 7.27-7.63(\mathrm{~m}, 9 \mathrm{H})$, 5.17-5.25 (m, 2H), 2.49-2.61 (m, 2H); ${ }^{13}$ C NMR (125 MHz, DMSO-d6) \$168.0, 161.7, 159.7, 138.1, 131.7, 131.6, 130.2, 129.9, 129.8, $128.9,127.0,126.9,126.0,116.9,116.8,51.9,45.2,36.3$. IR (KBr): 3213, 1645, 1597, 1590, 1492, 1455, 1312, $1234 \mathrm{~cm}^{-1}$. MS (ESI) m/z $287\left([\mathrm{M}+\mathrm{H}]^{+}\right)$. 

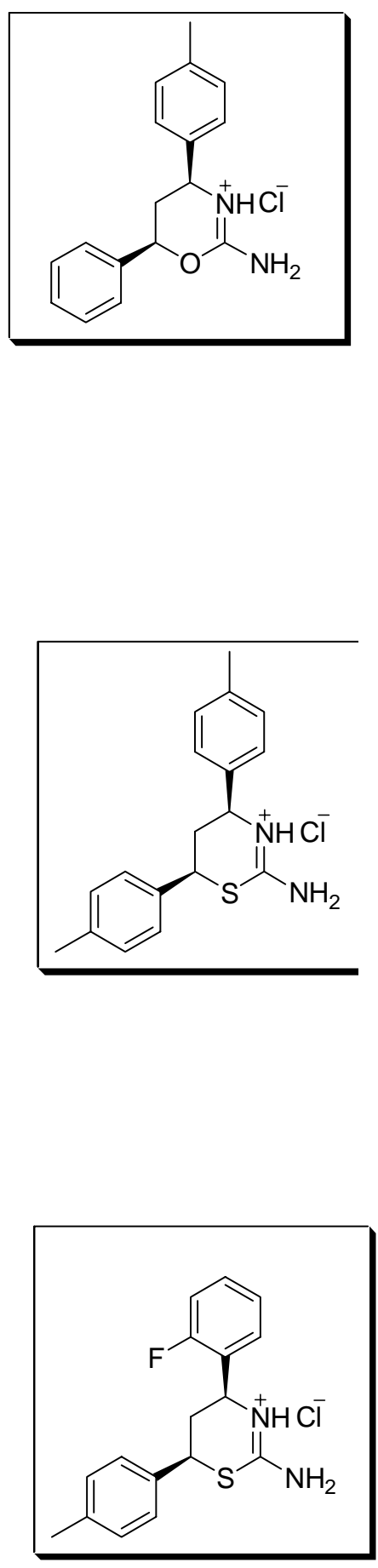

4f: m.p. $207-209{ }^{\circ} \mathrm{C} .{ }^{\mathbf{1}} \mathbf{H}$ NMR $\left(500 \mathrm{MHz}, \mathrm{DMSO}-d_{6}\right) \delta 9.34$ (br s, 2H), $7.63(\mathrm{~d}, \mathrm{~J}=8.5 \mathrm{~Hz}, 2 \mathrm{H}), 7.41(\mathrm{~m}, 5 \mathrm{H}), 7.40(\mathrm{~d}, \mathrm{~J}=8 \mathrm{~Hz}, 2 \mathrm{H})$, $6.30(\mathrm{~d}, \mathrm{~J}=3.5 \mathrm{~Hz}, 1 \mathrm{H}), 5.43(\mathrm{~d}, \mathrm{~J}=3.5 \mathrm{~Hz}, 1 \mathrm{H}), 2.34(\mathrm{~s}, 3 \mathrm{H}) ;{ }^{13} \mathbf{C}$ NMR $(125 \mathrm{MHz}$, DMSO-d6) $\delta$ 156.1, 144.6, 140.8, 140.3, 129.9, 129.6, 129.2, 127.6, 127.4, 124.8, 101.0, 52.4, 21.4. IR (KBr): 3224, 3114, 1673, 1550, 1517, 1457, $1113 \mathrm{~cm}^{-1}$. MS (ESI) m/z $267\left([\mathrm{M}+\mathrm{H}]^{+}\right)$.

4g: m.p. $273-276{ }^{\circ} \mathrm{C} .{ }^{1} \mathbf{H}$ NMR $\left(500 \mathrm{MHz}, \mathrm{DMSO}-d_{6}\right) \delta 10.67$ (s, 1H), $9.78(\mathrm{~s}, 1 \mathrm{H}), 9.10(\mathrm{~s}, 1 \mathrm{H}), 7.21-7.42(\mathrm{~m}, 8 \mathrm{H}), 5.06(\mathrm{t}, 1 \mathrm{H})$, $4.90(\mathrm{t}, 1 \mathrm{H}), 2.42(\mathrm{~m}, 2 \mathrm{H}), 2.29-2.39(\mathrm{~d}, 6 \mathrm{H}) ;{ }^{13} \mathbf{C}$ NMR (125 MHz, DMSO-d6) $\delta 167.1,138.3,137.8,136.4,134.3,129.6,129.3,129.1$, 127.7, 126.8, 126.0, 113.1, 56.5, 44.3, 37.4, 20.7. IR (KBr): 3218 , 2798, 1633, 1597, 1514, 1485, $1115 \mathrm{~cm}^{-1}$. MS (ESI) m/z 297 $\left([\mathrm{M}+\mathrm{H}]^{+}\right)$.

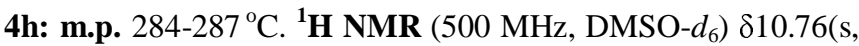
1H), $9.65(\mathrm{~s}, 1 \mathrm{H}), 9.07(\mathrm{~s}, 1 \mathrm{H}), 7.22-7.67(\mathrm{~m}, 8 \mathrm{H}), 5.14(\mathrm{t}, 1 \mathrm{H})$, $5.12(\mathrm{t}, 1 \mathrm{H}), 2.48(\mathrm{~m}, 2 \mathrm{H}), 2.30(\mathrm{~s}, 3 \mathrm{H}) ;{ }^{13} \mathrm{C}$ NMR (125 MHz, DMSO-d6) $\delta 167.1,160.7,158.7,138.4,134.1,130.6,129.7$, 128.8, 127.8, 126.0, 125.0, 115.9, 115.7, 50.9, 44.0, 35.4, 20.7. IR (KBr): 3201, 3022, 1639, 1596, 1493, 1454, 1333, 1232. MS (ESI) $\mathrm{m} / \mathrm{z} 301\left([\mathrm{M}+\mathrm{H}]^{+}\right)$. 
6a: m.p. $254-257{ }^{\circ}$ C. ${ }^{1} \mathbf{H}$ NMR (500 MHz, DMSO- $\left.d_{6}\right) \delta$ 7.26-7.15 (m, 5H), $6.51(\mathrm{~s}, 1 \mathrm{H}), 4.51(\mathrm{~d}, J=10.8 \mathrm{~Hz}, 1 \mathrm{H}), 4.42(\mathrm{q}$, $J=1.9 \mathrm{~Hz}, 1 \mathrm{H}), 3.89(\mathrm{~d}, J=9.3 \mathrm{~Hz}, 1 \mathrm{H}), 3.44(\mathrm{t}, J=9.9 \mathrm{~Hz}, 1 \mathrm{H})$, $2.29(\mathrm{~s}, 3 \mathrm{H}), 1.78-1.67(\mathrm{~m}, 2 \mathrm{H}), 1.55-1.50(\mathrm{~m}, 1 \mathrm{H}), 1.27-1.18(\mathrm{~m}$,

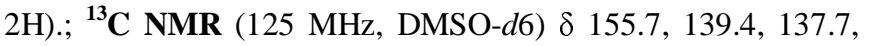
$129.8,128.3,81.2,66.7,53.3,38.7,23.8,21.6,21.3 . \mathbf{I R}(\mathrm{KBr})$ : 3284, 1690, 1502, 1477, 1183, $1130 \mathrm{~cm}^{-1}$. MS (ESI) m/z 247 $\left([\mathrm{M}+\mathrm{H}]^{+}\right)$.

6b: m.p. $215-217{ }^{\circ}$ C. ${ }^{1} \mathbf{H}$ NMR (500 MHz, DMSO- $\left.d_{6}\right) \quad \delta$ 7.37-7.28 (m, 6H), $6.58(\mathrm{~s}, 1 \mathrm{H}), 4.55(\mathrm{~d}, J=10.8 \mathrm{~Hz}, 1 \mathrm{H})$, $4.42(\mathrm{~s}, 1 \mathrm{H}), 3.90(\mathrm{~d}, J=10.1 \mathrm{~Hz}, 1 \mathrm{H}), 3.44(\mathrm{t}, J=10.6 \mathrm{~Hz}$ 1H), 1.81-1.70 (m, 2H), 1.56-1.51 (m, 1H), 1.26-1.19 (m, 2H); ${ }^{13}$ C NMR (125 MHz, DMSO-d6) $\delta 155.6,142.5,129.3,128.6$, $128.4,81.2,66.7,53.6,38.7,23.8,21.3$. IR (KBr): 3432, 2921, 1685, 1658, $1510 \mathrm{~cm}^{-1}$. MS (ESI) m/z $233\left([\mathrm{M}+\mathrm{H}]^{+}\right)$.

6c: m.p. $221-224{ }^{\circ} \mathrm{C} .{ }^{\mathbf{1}} \mathbf{H}$ NMR $\left(500 \mathrm{MHz}, \mathrm{DMSO}-d_{6}\right) \delta 7.27$ (s, $1 \mathrm{H}), 7.25(\mathrm{~d}, J=8.0 \mathrm{~Hz}, 2 \mathrm{H}), 6.92(\mathrm{~d}, J=8.0 \mathrm{~Hz}, 2 \mathrm{H}), 6.50(\mathrm{~s}$, $1 \mathrm{H}), 4.51(\mathrm{~d}, J=10.9 \mathrm{~Hz}, 1 \mathrm{H}), 4.42(\mathrm{~s}, 1 \mathrm{H}), 3.88(\mathrm{~d}, J=8.1 \mathrm{~Hz}$, $1 \mathrm{H}), 3.44(\mathrm{t}, J=11.2 \mathrm{~Hz}, 1 \mathrm{H}), 1.78-1.67(\mathrm{~m}, 2 \mathrm{H}), 1.55-1.50$ $(\mathrm{m}, 1 \mathrm{H}), 1.27-1.17(\mathrm{~m}, 2 \mathrm{H}) ;{ }^{13} \mathbf{C}$ NMR (125 MHz, DMSO-d6) $\delta$ $159.7,155.7,134.2,129.5,129.3,114.7,81.3,66.7,56.0$, 52.9, 38.8, 23.8, 21.3. IR (KBr): 3266, 1684, 1612, 1506, 1169, $1083 \mathrm{~cm}^{-1}$. MS (ESI) m/z $263\left([\mathrm{M}+\mathrm{H}]^{+}\right)$. 
6d: m.p. $273-276{ }^{\circ} \mathrm{C} .{ }^{1} \mathbf{H}$ NMR (500 MHz, DMSO- $\left.d_{6}\right) \delta$ $7.42-7.32(\mathrm{~m}, 5 \mathrm{H}), 6.67(\mathrm{~s}, 1 \mathrm{H}), 4.57(\mathrm{~d}, J=10.8 \mathrm{~Hz}, 1 \mathrm{H})$, $4.43(\mathrm{~d}, J=1.7 \mathrm{~Hz}, 1 \mathrm{H}), 3.89(\mathrm{~d}, J=9.6 \mathrm{~Hz}, 1 \mathrm{H}), 3.44(\mathrm{t}, J=$ $11.4 \mathrm{~Hz}, 1 \mathrm{H}), 1.80-1.72(\mathrm{~m}, 2 \mathrm{H}), 1.58-1.55$ (m, $1 \mathrm{H}), 1.24-1.19$ $(\mathrm{m}, 2 \mathrm{H}) ;{ }^{13} \mathrm{C}$ NMR (125 MHz, DMSO-d6) $\delta$ 155.6, 141.5, $133.0,130.4,129.3,81.2,66.7,53.0,38.6,23.8,21.2 .$. IR (KBr): 3306, 3242, 1697, 1489, 1210, $1182 \mathrm{~cm}^{-1} . \mathbf{M S}(\mathrm{ESI}) \mathrm{m} / \mathrm{z}$ $267\left([\mathrm{M}+\mathrm{H}]^{+}\right)$.

6e: m.p. $236-238{ }^{\circ} \mathrm{C} .{ }^{1} \mathbf{H}$ NMR $\left(500 \mathrm{MHz}, \mathrm{DMSO}-d_{6}\right) \delta 8.81(\mathrm{~d}$, $J=2.5 \mathrm{~Hz}, 1 \mathrm{H}), 8.38(\mathrm{~s}, 1 \mathrm{H}), 7.42-7.22(\mathrm{~m}, 5 \mathrm{H}), 4.51(\mathrm{~d}, J=$ $4.1 \mathrm{~Hz}, 1 \mathrm{H}), 4.40(\mathrm{t}, J=2.8 \mathrm{~Hz}, 1 \mathrm{H}), 3.88(\mathrm{~d}, J=11.3 \mathrm{~Hz}, 1 \mathrm{H})$, $3.47(\mathrm{t}, J=10.3 \mathrm{~Hz}, 1 \mathrm{H}), 1.88-1.86(\mathrm{~m}, 1 \mathrm{H}), 1.76-1.59(\mathrm{~m}$, 2H), $1.26(\mathrm{~d}, J=10.4 \mathrm{~Hz}, 1 \mathrm{H}) ;{ }^{13} \mathbf{C}$ NMR (125 MHz, DMSO-d6) $\delta 177.5,141.1,129.4,128.8,128.4,79.2,66.4,54.9,37.0$, 23.5, 21.4. IR (KBr): 3432, 3171, 2970, 1536, $1406 \mathrm{~cm}^{-1} . \mathbf{M S}$ (ESI) $\mathrm{m} / \mathrm{z} 249\left([\mathrm{M}+\mathrm{H}]^{+}\right)$.

6f: m.p. $232-235{ }^{\circ} \mathrm{C} .{ }^{\mathbf{1}} \mathbf{H}$ NMR $\left(500 \mathrm{MHz}, \mathrm{DMSO}-d_{6}\right) \delta 8.89$ (s, 1H), 8.48 (s, 1H), 7.47-7.19 (m, 4H), $4.82(\mathrm{~d}, J=10.8 \mathrm{~Hz}, 1 \mathrm{H})$, $4.43(\mathrm{~d}, J=1.3 \mathrm{~Hz}, 1 \mathrm{H}), 3.89(\mathrm{~d}, J=10.6 \mathrm{~Hz}, 1 \mathrm{H}), 3.47(\mathrm{t}, J=$ $9.7 \mathrm{~Hz}, 1 \mathrm{H}), 3.47(\mathrm{~d}, J=9.8 \mathrm{~Hz}, 2 \mathrm{H}), 1.67-1.23(\mathrm{~m}, 4 \mathrm{H}) ;{ }^{13} \mathrm{C}$ NMR (125 MHz, DMSO-d6) $\delta$ 177.6, 162.2, 160.3, 130.8, $130.7,130.2,127.7,127.6,125.8,116.4,116.2,79.2,66.6$, 48.3, 36.1, 23.7, 21.5. IR (KBr): 3175, 2971, 1573, 1532, 1491, 1253, 1203, $1036 \mathrm{~cm}^{-1}$. MS (ESI) m/z $267\left([\mathrm{M}+\mathrm{H}]^{+}\right)$. 
6g: m.p. $267-269{ }^{\circ} \mathrm{C} .{ }^{1} \mathbf{H}$ NMR (500 Mz, DMSO-d $\left.d_{6}\right) \delta 8.79$ (s, $1 \mathrm{H}), 8.31(\mathrm{~s}, 1 \mathrm{H}), 7.17(\mathrm{~m}, 4 \mathrm{H}), 4.46(\mathrm{~d}, J=10.4 \mathrm{~Hz}, 1 \mathrm{H})$, $4.38(\mathrm{~s}, 1 \mathrm{H}), 3.87(\mathrm{~d}, J=11.0 \mathrm{~Hz}, 1 \mathrm{H}), 3.47(\mathrm{t}, J=11.0 \mathrm{~Hz}$, $1 \mathrm{H}), 2.30(\mathrm{~s}, 3 \mathrm{H}), 1.84-1.57(\mathrm{~m}, 3 \mathrm{H}), 1.26(\mathrm{~d}, J=7.4 \mathrm{~Hz}, 1 \mathrm{H})$; ${ }^{13}$ C NMR (125 Mz, DMSO- $\left.d_{6}\right) \delta 177.4,138.1,137.9,129.9$, 128.3, 79.2, 66.4, 54.6, 37.0, 23.5, 21.7, 21.4. IR (KBr): 3196, $2953,2859,1572,1536,1513,1203,1036 \mathrm{~cm}^{-1} . \mathbf{M S}(\mathrm{ESI}) \mathrm{m} / \mathrm{z}$ $263\left([\mathrm{M}+\mathrm{H}]^{+}\right)$.

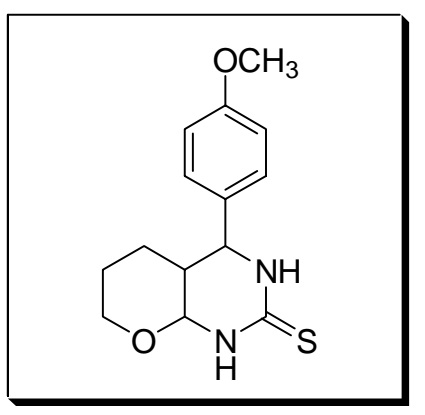

6h: m.p. $246-249{ }^{\circ}$ C. ${ }^{\mathbf{1}} \mathbf{H}$ NMR $\left(500 \mathrm{Mz}, \mathrm{DMSO}-d_{6}\right) \delta 8.79(\mathrm{~d}, J=$ $2.2 \mathrm{~Hz}, 1 \mathrm{H}), 8.18(\mathrm{~s}, 1 \mathrm{H}), 7.23(\mathrm{~d}, J=8.3 \mathrm{~Hz}, 1 \mathrm{H}), 6.94(\mathrm{~d}, J=$ $8.3 \mathrm{~Hz}, 1 \mathrm{H}), 4.47(\mathrm{~d}, J=10.6 \mathrm{~Hz}, 1 \mathrm{H}), 4.40(\mathrm{~s}, 1 \mathrm{H}), 3.88(\mathrm{~d}, J=$ $10.9 \mathrm{~Hz}, 1 \mathrm{H}), 3.75(\mathrm{~s}, 1 \mathrm{H}), 3.46(\mathrm{t}, J=11.0 \mathrm{~Hz}, 1 \mathrm{H}), 1.85-1.57$ $(\mathrm{m}, 3 \mathrm{H}), 1.26-1.21(\mathrm{~m}, 2 \mathrm{H}) ;{ }^{13} \mathrm{C}$ NMR (125 Mz, DMSO- $\left.d_{6}\right) \delta$ $177.3,159.8,132.8,129.6,114.7,79.3,66.6,56.0,54.2,37.0$, 23.5, 21.3. IR (KBr): 3179, 2976, 2945, 1612, 1561, 1463, 1249, $1050 \mathrm{~cm}^{-1}$. MS (ESI) m/z $279\left([\mathrm{M}+\mathrm{H}]^{+}\right)$.

6i: m.p. $255-257{ }^{\circ}$ C. ${ }^{1} \mathbf{H}$ NMR $\left(500 \mathrm{Mz}\right.$, DMSO- $\left.d_{6}\right) \delta 8.84(\mathrm{~d}, J$ $=2.0 \mathrm{~Hz}, 1 \mathrm{H}), 8.42(\mathrm{~s}, 1 \mathrm{H}), 7.37-7.16(\mathrm{~m}, 4 \mathrm{H}), 4.54(\mathrm{~d}, J=$ $10.6 \mathrm{~Hz}, 1 \mathrm{H}), 4.40(\mathrm{~s}, 1 \mathrm{H}), 3.88(\mathrm{~d}, J=11.2 \mathrm{~Hz}, 1 \mathrm{H}), 3.47(\mathrm{t}, J$ $=11.0 \mathrm{~Hz}, 1 \mathrm{H}), 1.88-1.59(\mathrm{~m}, 3 \mathrm{H}), 1.24(\mathrm{~d}, J=10.9 \mathrm{~Hz}, 2 \mathrm{H})$; ${ }^{13}$ C NMR (125 Mz, DMSO- $\left.d_{6}\right) \delta 177.4,163.6,161.7,137.2$, $130.6,130.5,116.2,116.0,79.2,66.6,54.0,37.0,23.4,21.3$. IR (KBr): 3176, 2976, 2861, 1604, 1575, 1539, 1508, 1207, $1032 \mathrm{~cm}^{-1}$. MS (ESI) m/z $267\left([\mathrm{M}+\mathrm{H}]^{+}\right)$. 
6j: m.p. $205-207{ }^{\circ} \mathrm{C} .{ }^{1} \mathbf{H}$ NMR $\left(500 \mathrm{Mz}\right.$, DMSO- $\left.d_{6}\right) \delta(\mathrm{ppm})$ $8.73(\mathrm{~s}, 1 \mathrm{H}), 8.22(\mathrm{~s}, 1 \mathrm{H}), 7.30-6.97(\mathrm{~m}, 4 \mathrm{H}), 4.88(\mathrm{~d}, J=$ $9.4 \mathrm{~Hz}, 1 \mathrm{H}), 4.39(\mathrm{t}, J=2.9 \mathrm{~Hz}, 1 \mathrm{H}), 3.80(\mathrm{~s}, 3 \mathrm{H}), 3.45(\mathrm{t}, J=$ 9.5Hz, 1H), 1.89-1.57 (m, 3H), 1.27 (s, 2H); ${ }^{13}$ C NMR (125 Mz, DMSO- $\left.d_{6}\right) \delta 177.1,157.4,129.3,128.7,128.0,121.2$, 111.6, 78.7, 65.3, 56.1, 35.8, 23.4, 21.6. IR (KBr): 3201, 2948, 2858, 1603, 1573, 1530, 1197, $1032 \mathrm{~cm}^{-1}$. MS (ESI) m/z 279 $\left([\mathrm{M}+\mathrm{H}]^{+}\right)$.

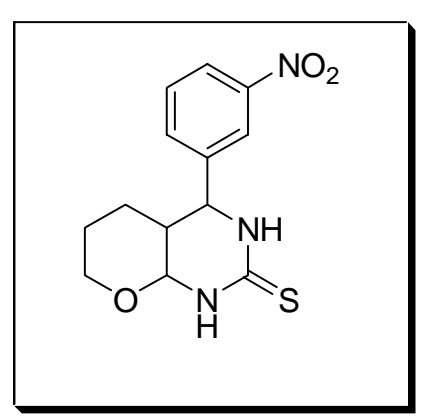

6k: m.p. $299-301{ }^{\circ} \mathrm{C} .{ }^{\mathbf{1}} \mathbf{H}$ NMR (500 Mz, DMSO- $\left.d_{6}\right) \delta(\mathrm{ppm})$ $8.95(\mathrm{~s}, 1 \mathrm{H}), 8.63(\mathrm{~s}, 1 \mathrm{H}), 8.19(\mathrm{~s}, 2 \mathrm{H}), 7.82-7.67(\mathrm{~m}, 2 \mathrm{H})$ $4.75(\mathrm{~d}, J=10.5 \mathrm{~Hz}, 1 \mathrm{H}), 4.44(\mathrm{~s}, 1 \mathrm{H}), 3.90(\mathrm{~d}, J=10.7 \mathrm{~Hz}$ $1 \mathrm{H}), 3.49(\mathrm{t}, J=10.7 \mathrm{~Hz}, 1 \mathrm{H}), 2.00-1.63(\mathrm{~m}, 3 \mathrm{H}), 1.25(\mathrm{t}, J=$ $13.3 \mathrm{~Hz}, 2 \mathrm{H}) ;{ }^{13} \mathbf{C}$ NMR $\left(125 \mathrm{Mz}, \mathrm{DMSO}-d_{6}\right) \delta 177.6,148.8$, $143.5,135.5,130.9,123.8,123.3,79.1,66.6,54.1,36.8,23.4$, 21.3. IR (KBr): 3203, 3082, 2839, 1566, 1526, 1348 1254, 1196 $\mathrm{cm}^{-1}$. MS (ESI) m/z $294\left([\mathrm{M}+\mathrm{H}]^{+}\right)$. 

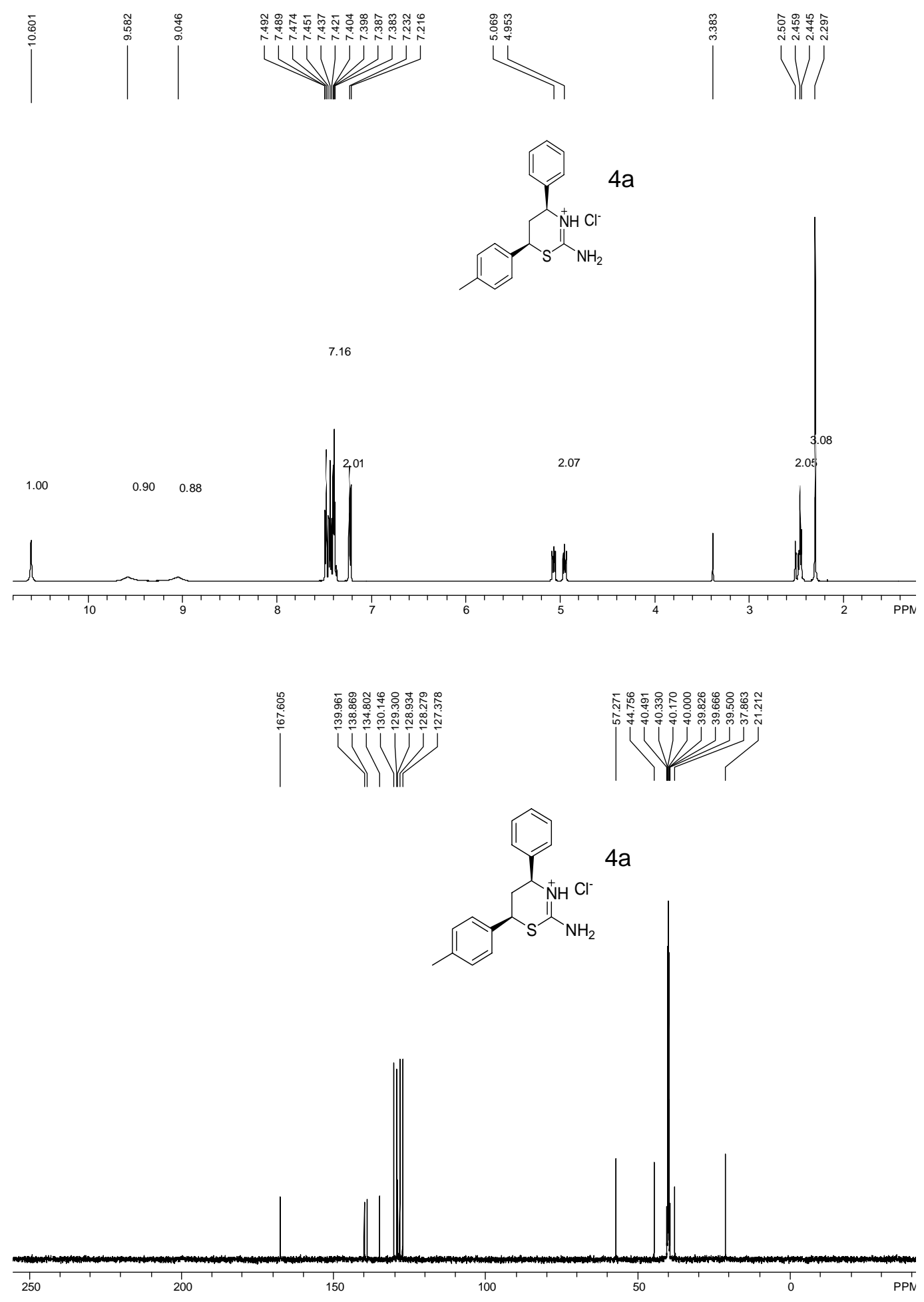

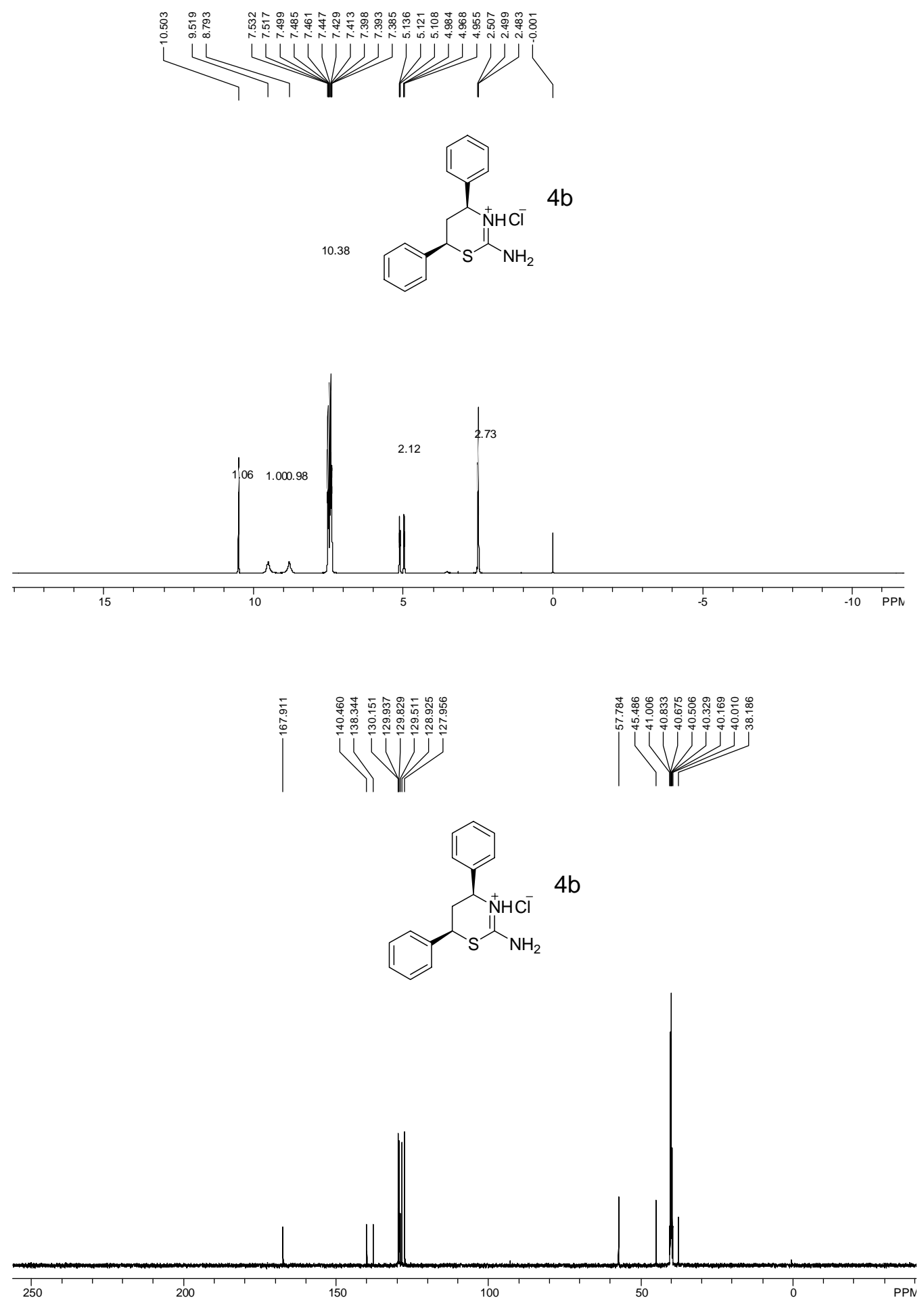

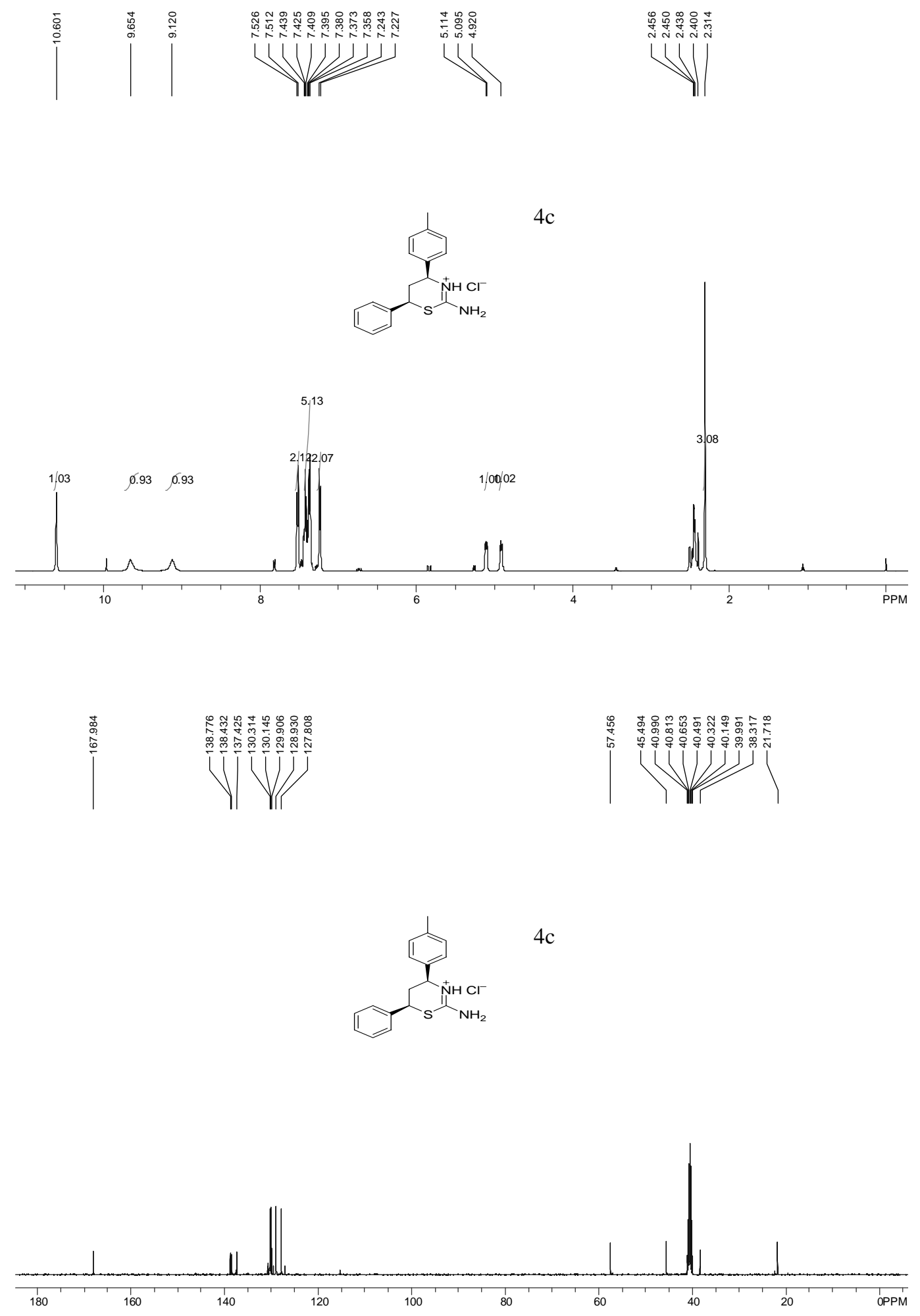

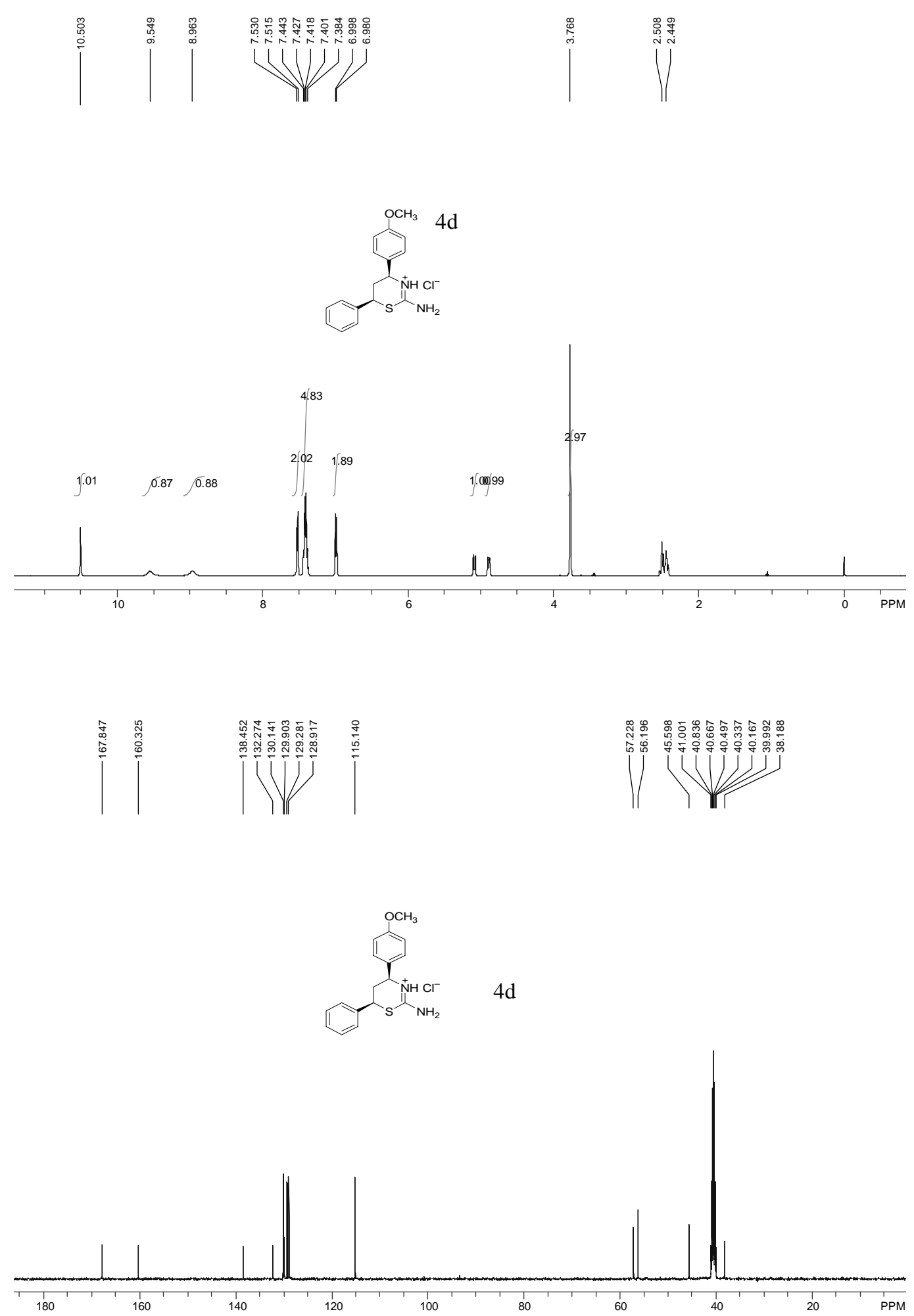

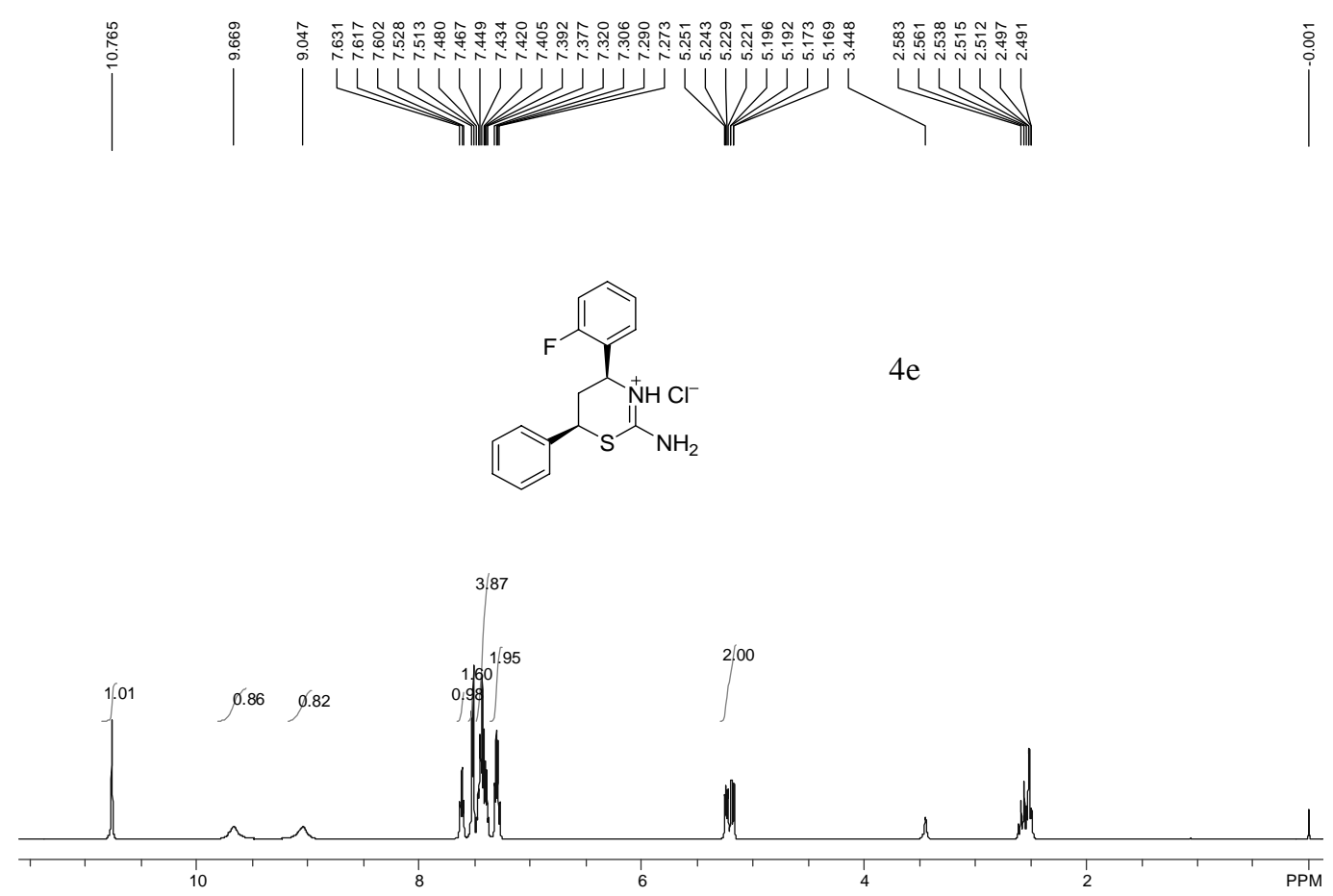
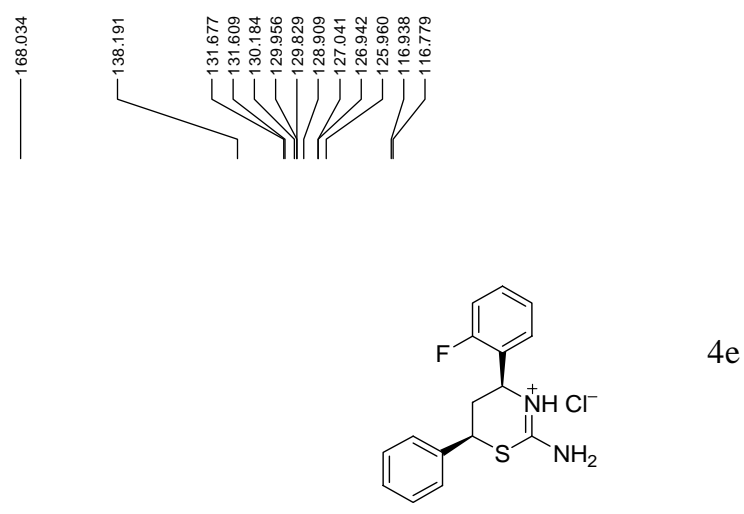

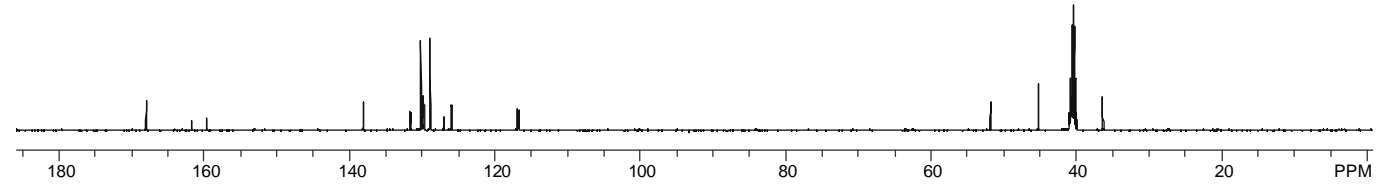



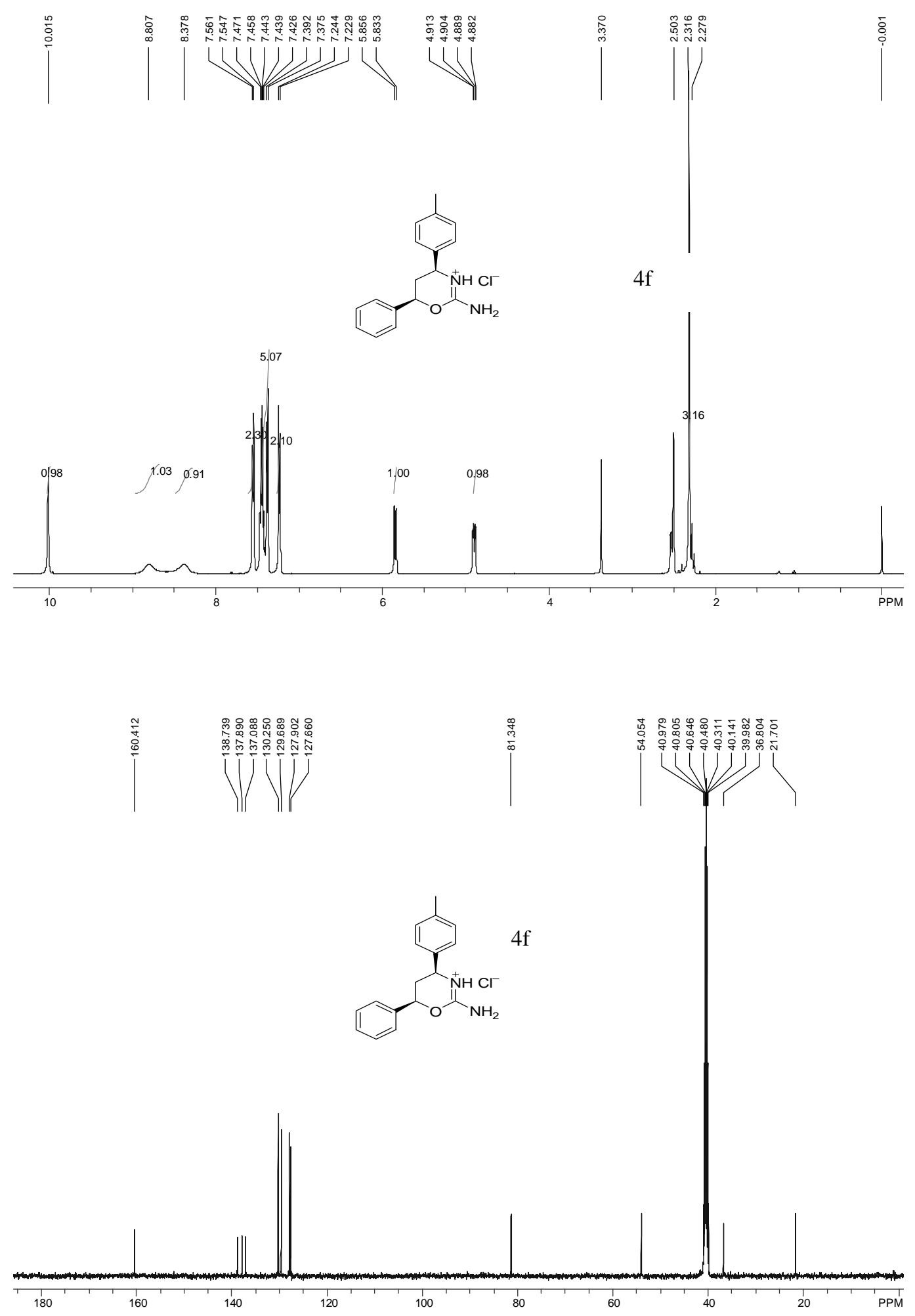

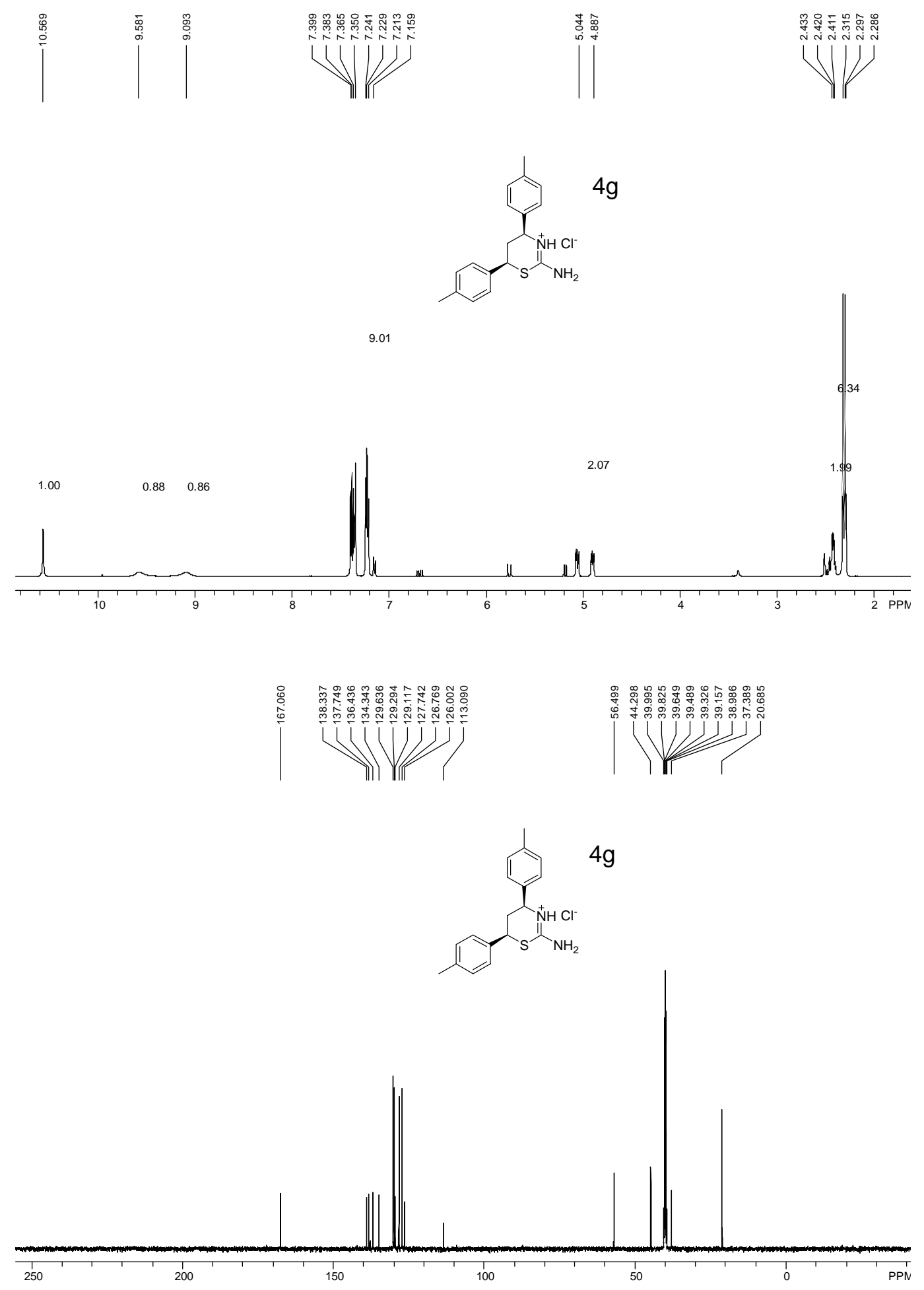

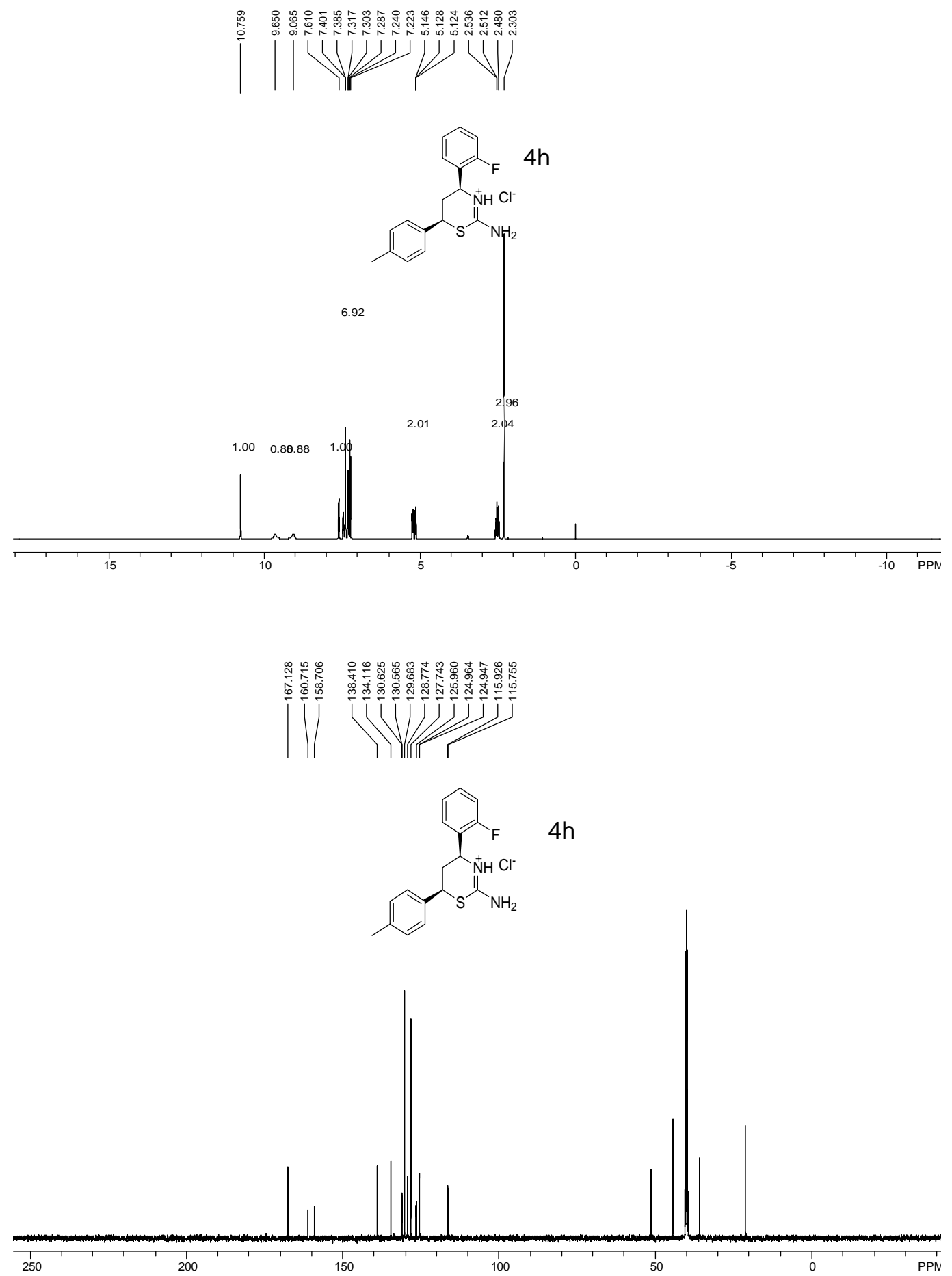

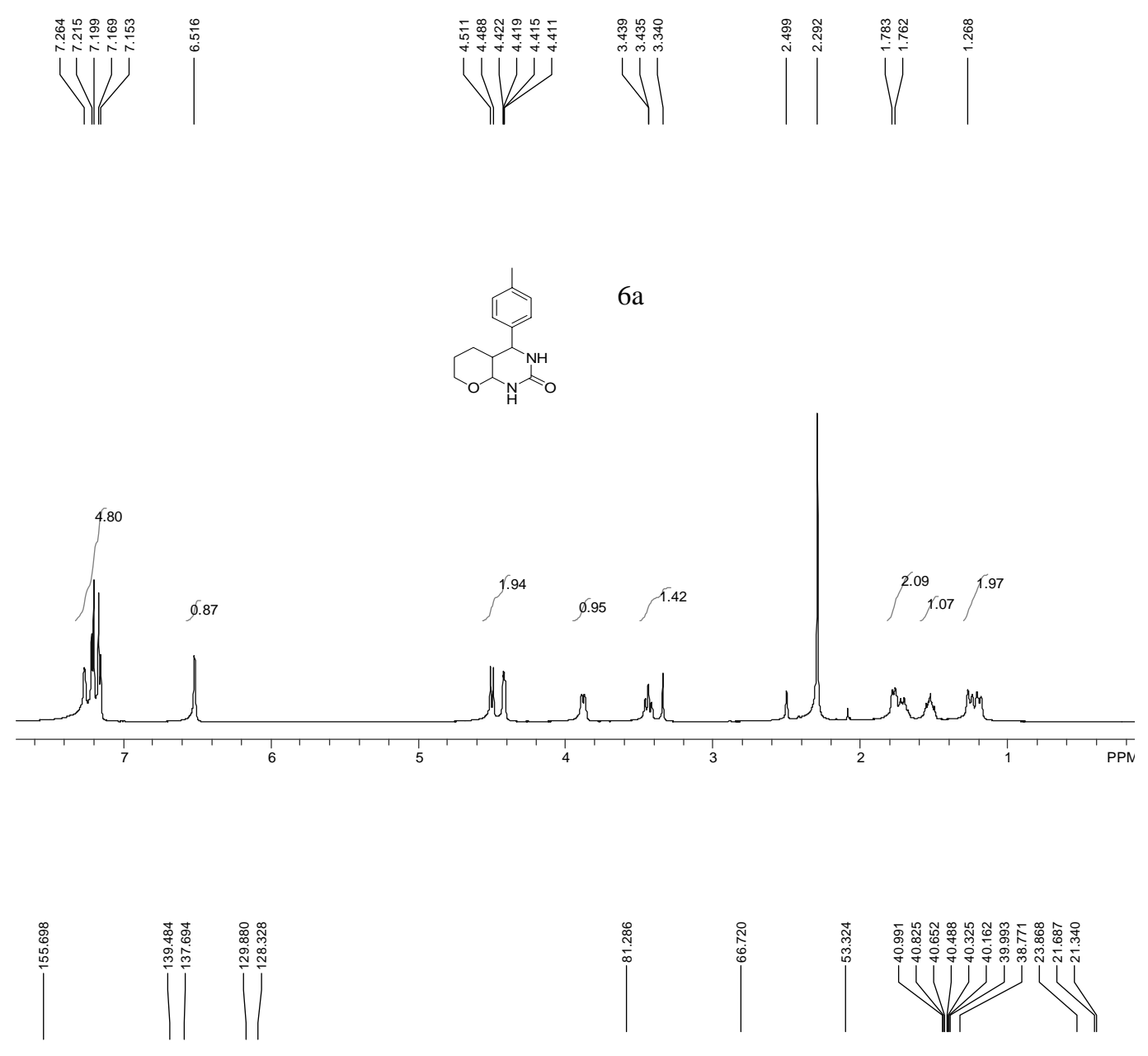

$6 a$

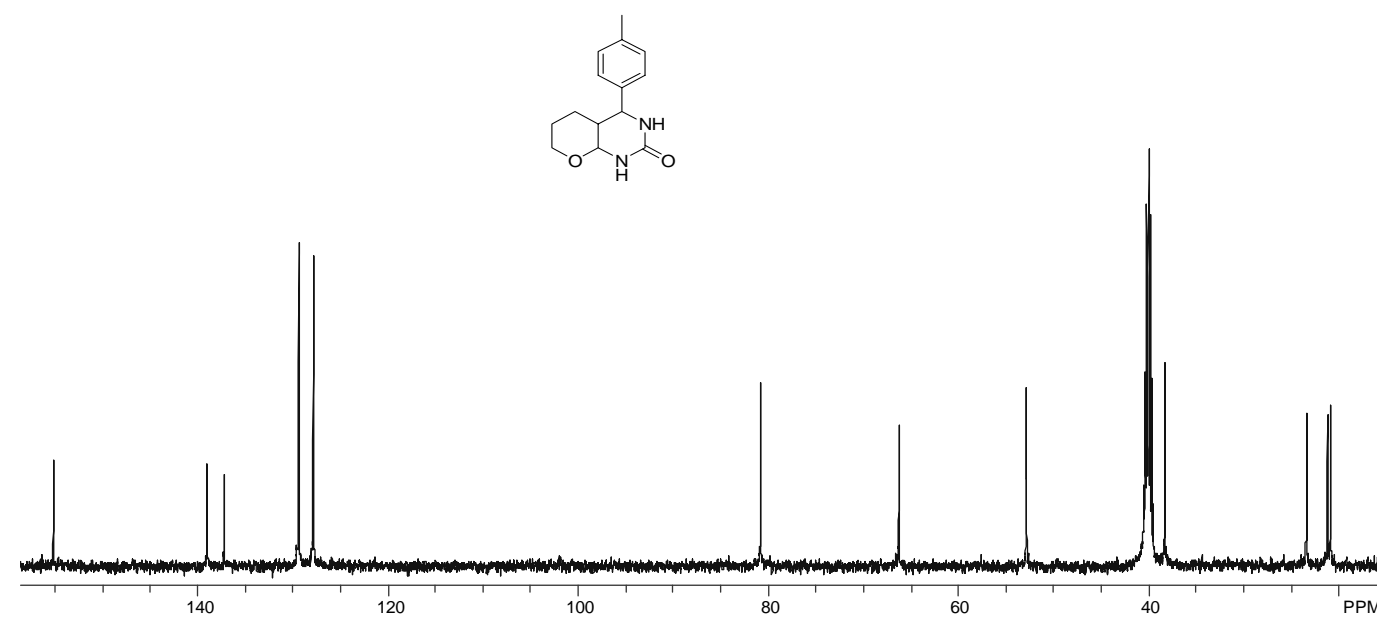



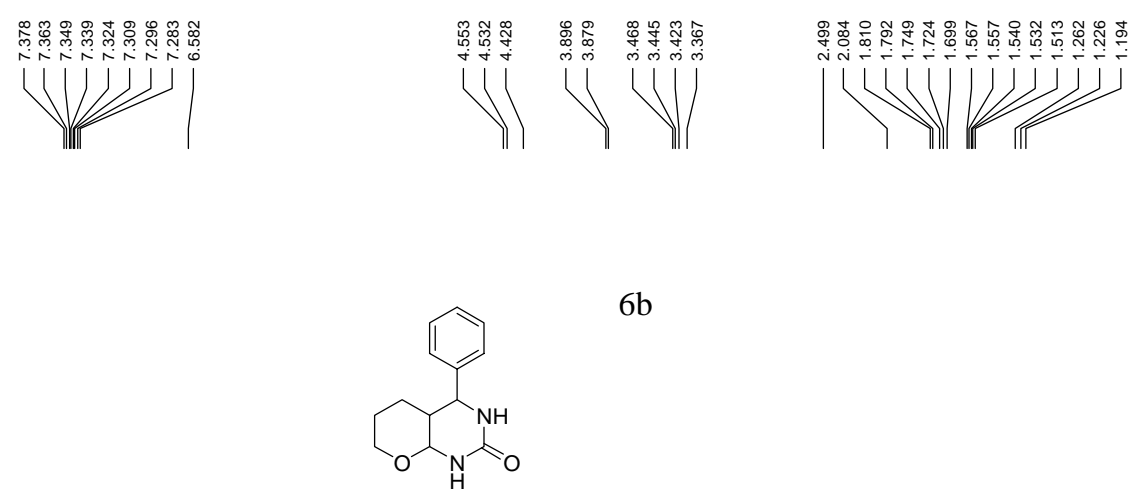

$6 b$
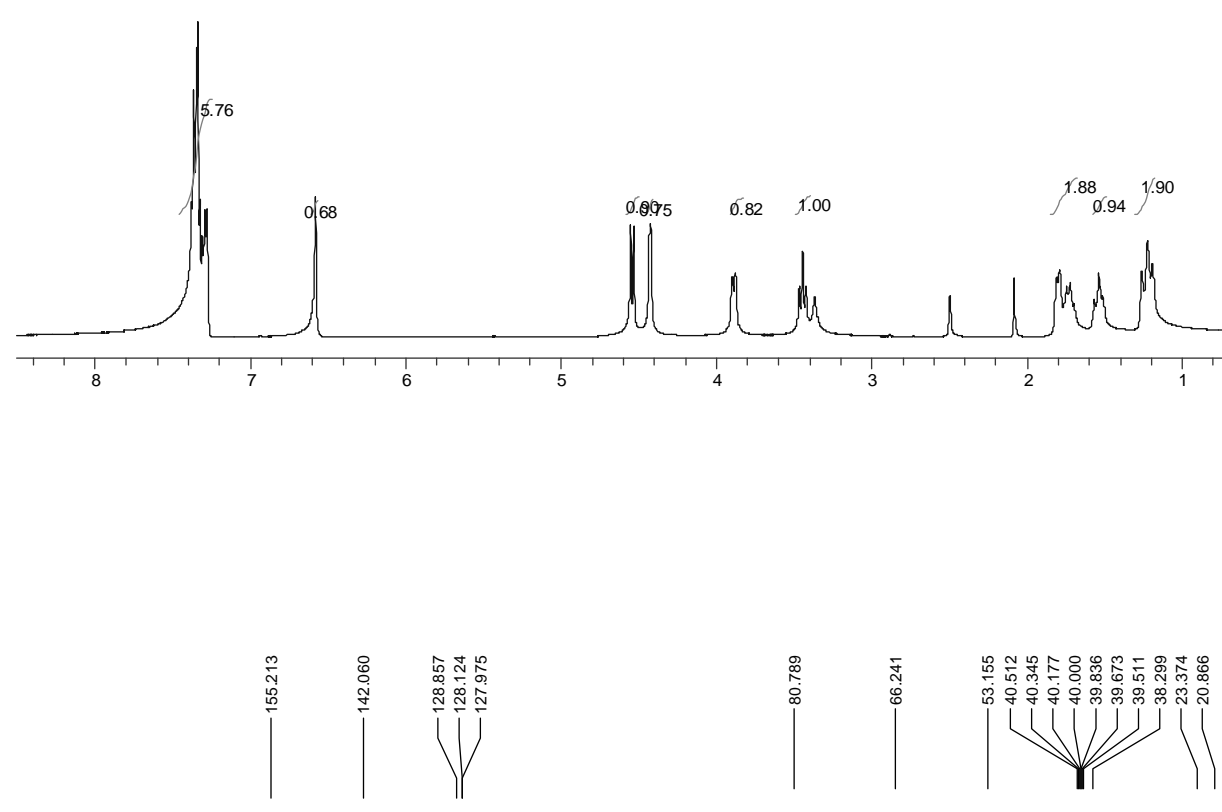

$6 b$<smiles>O=C1NC2OCCCC2C(c2ccccc2)N1</smiles>

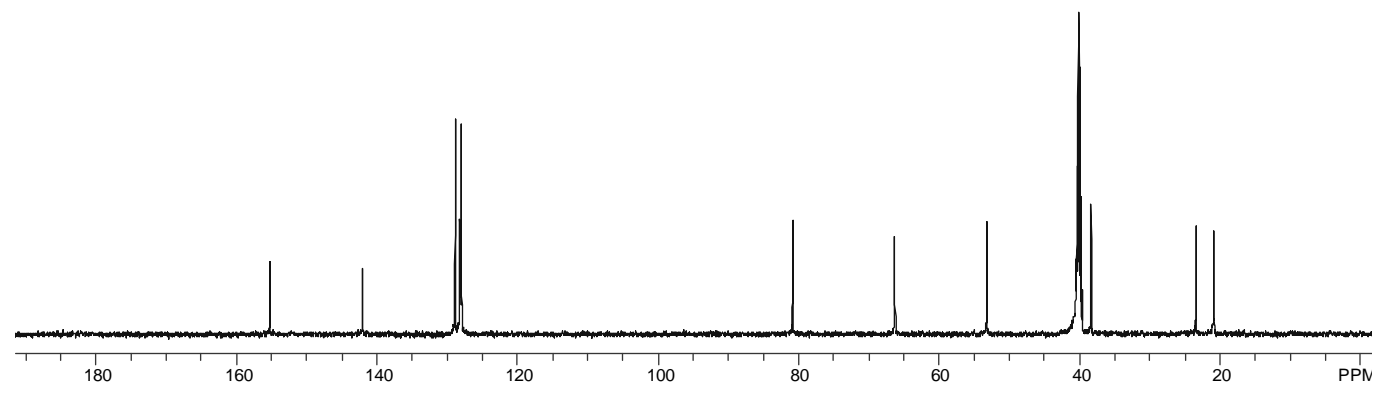



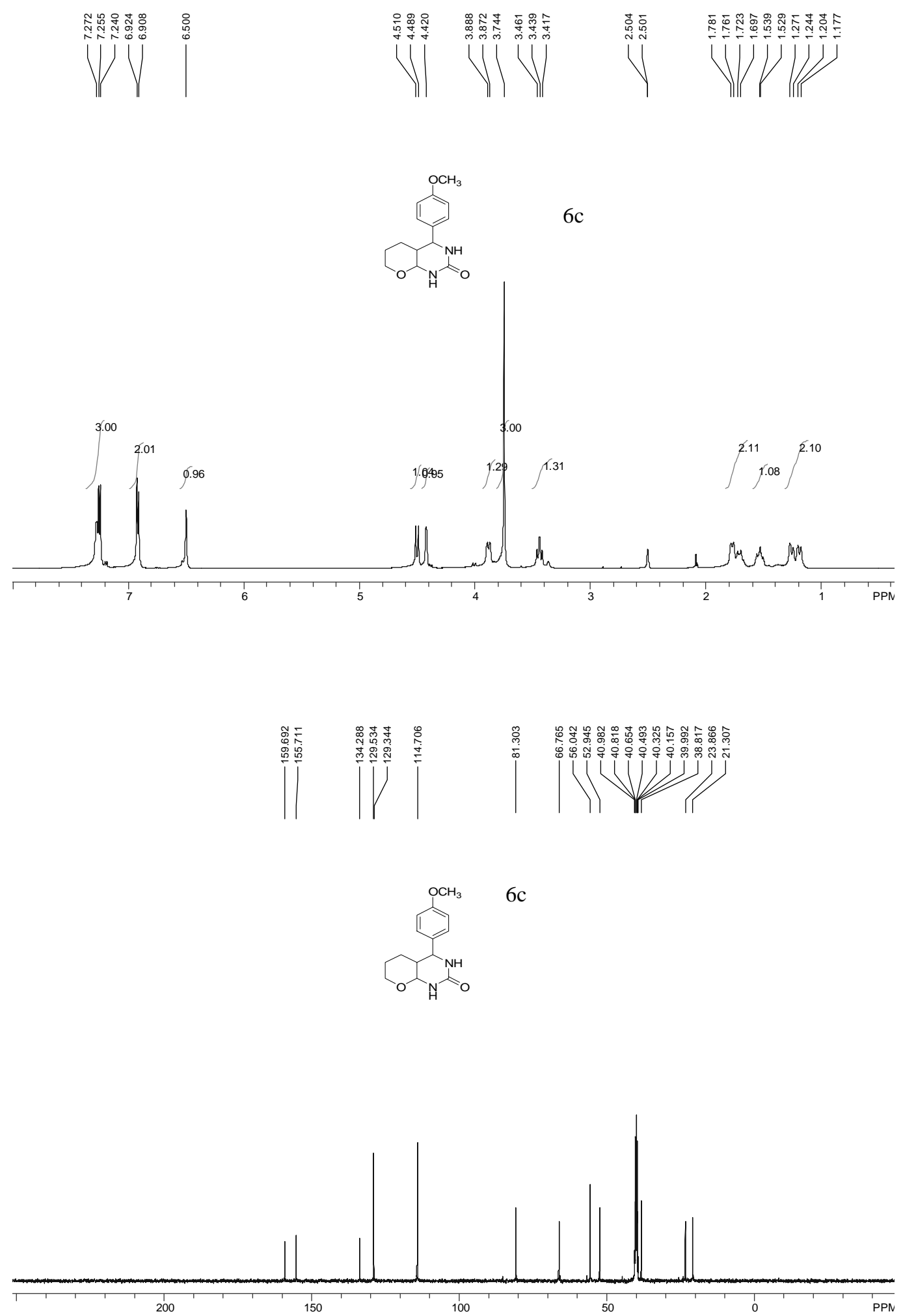

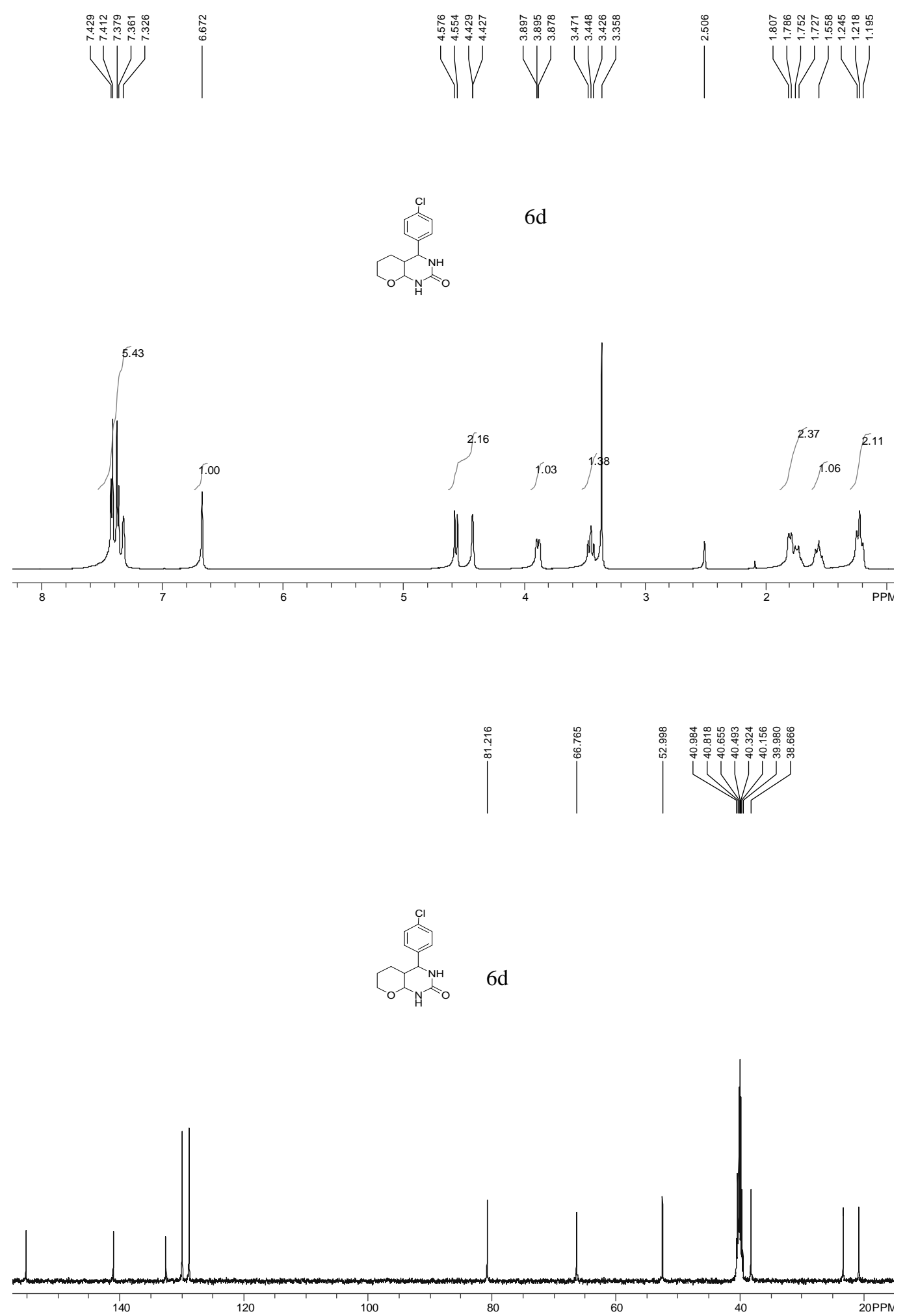


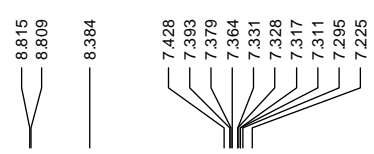

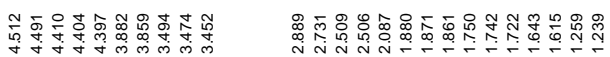

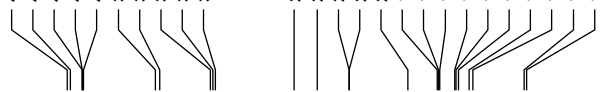

$6 e$
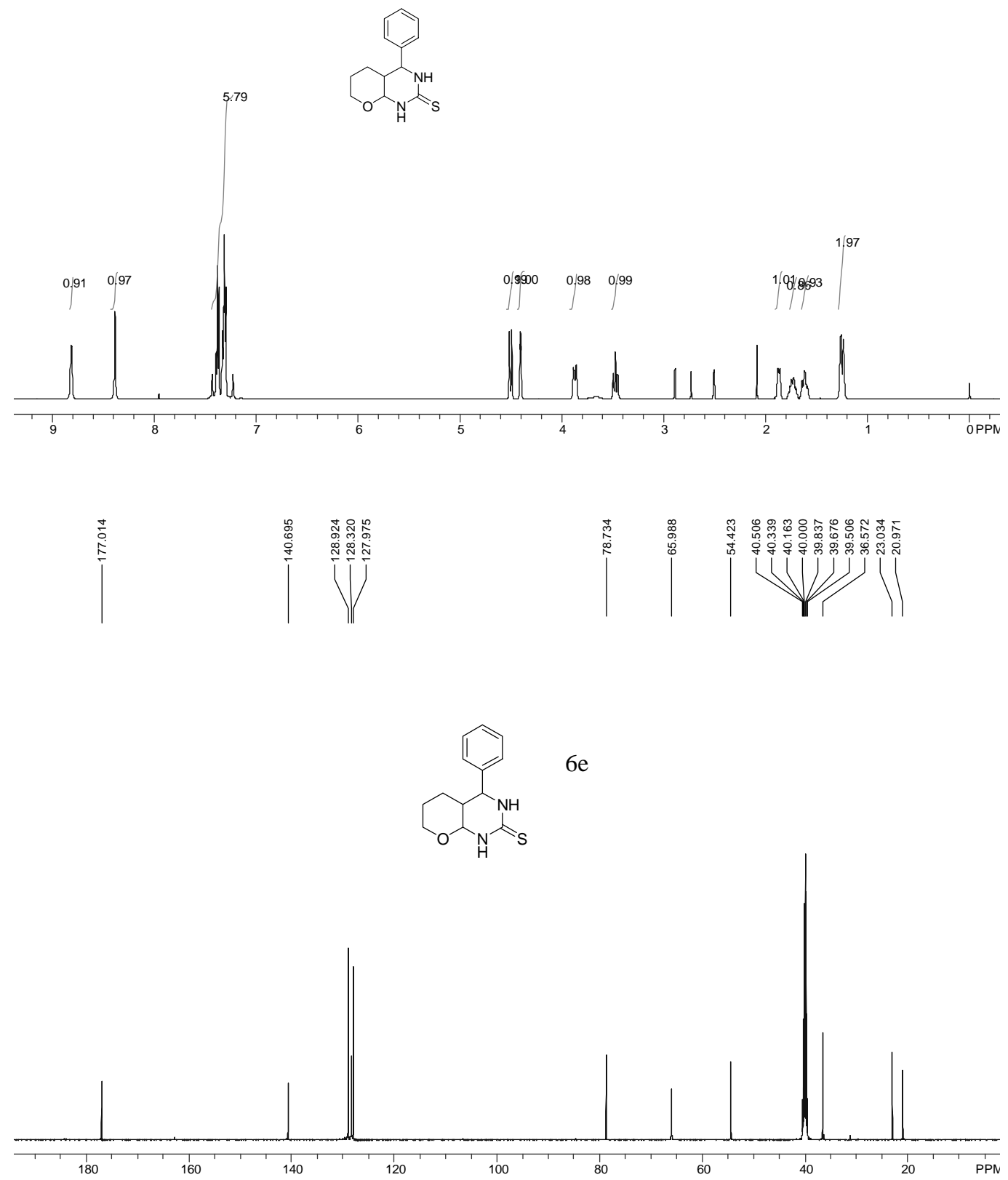

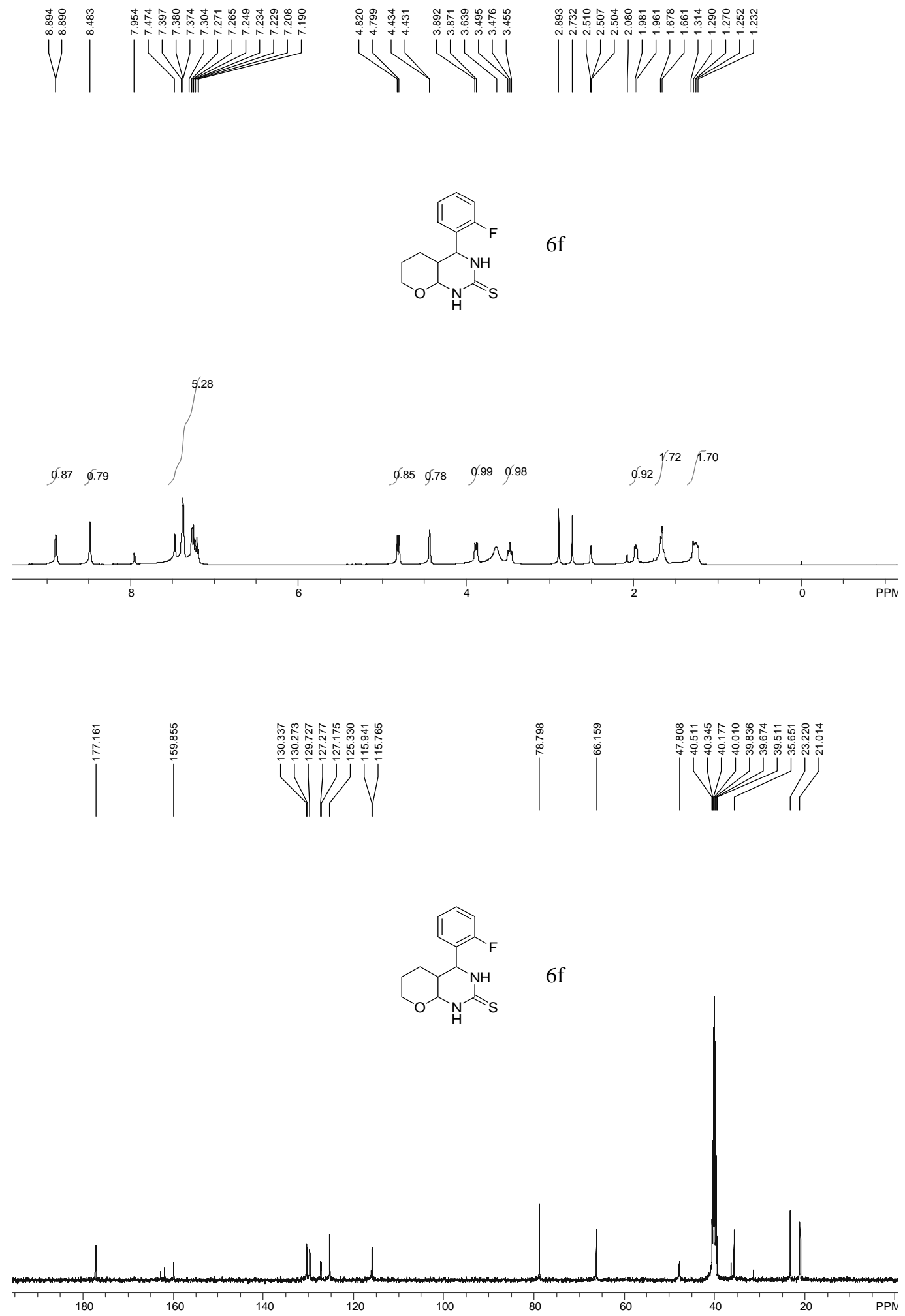

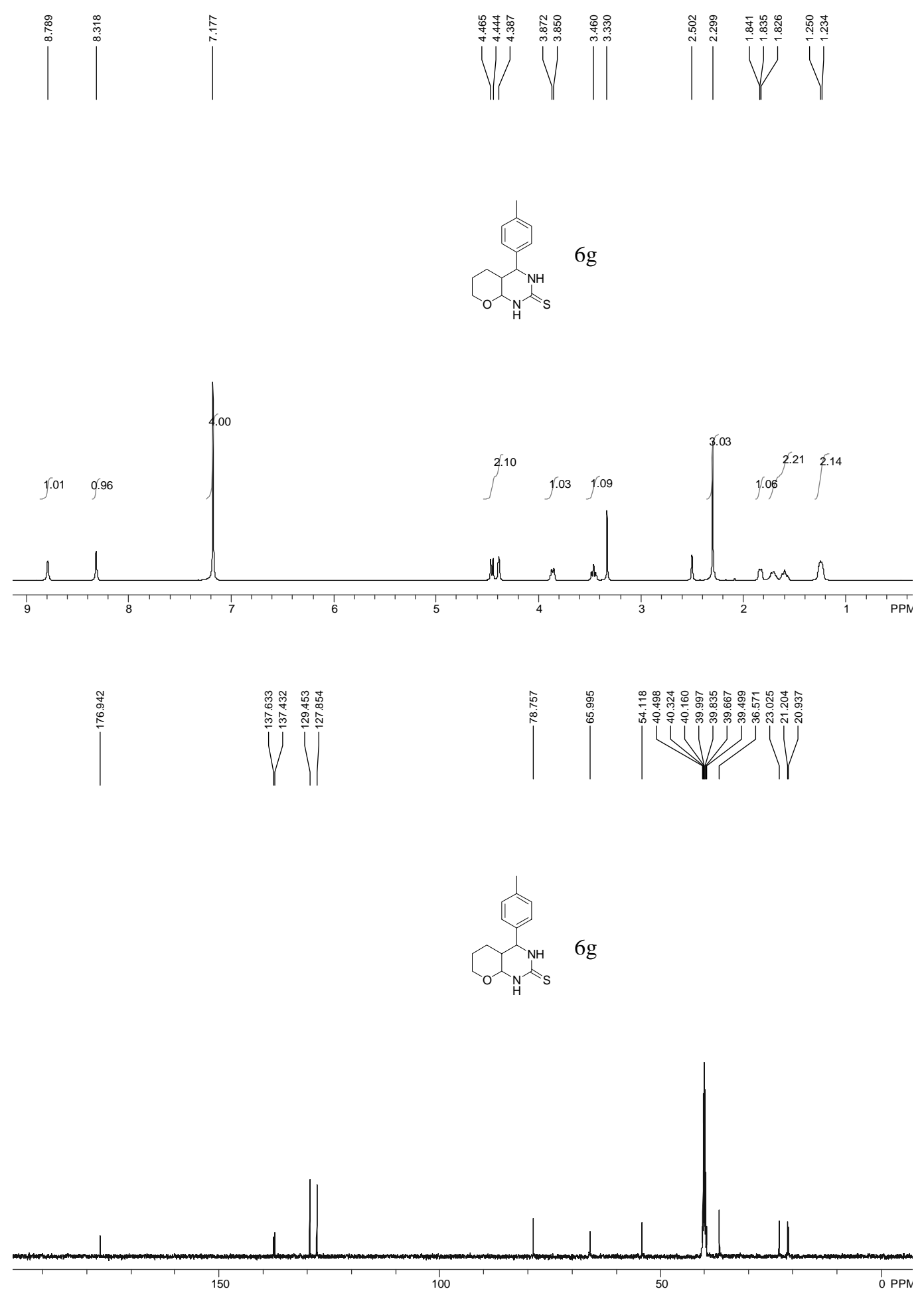

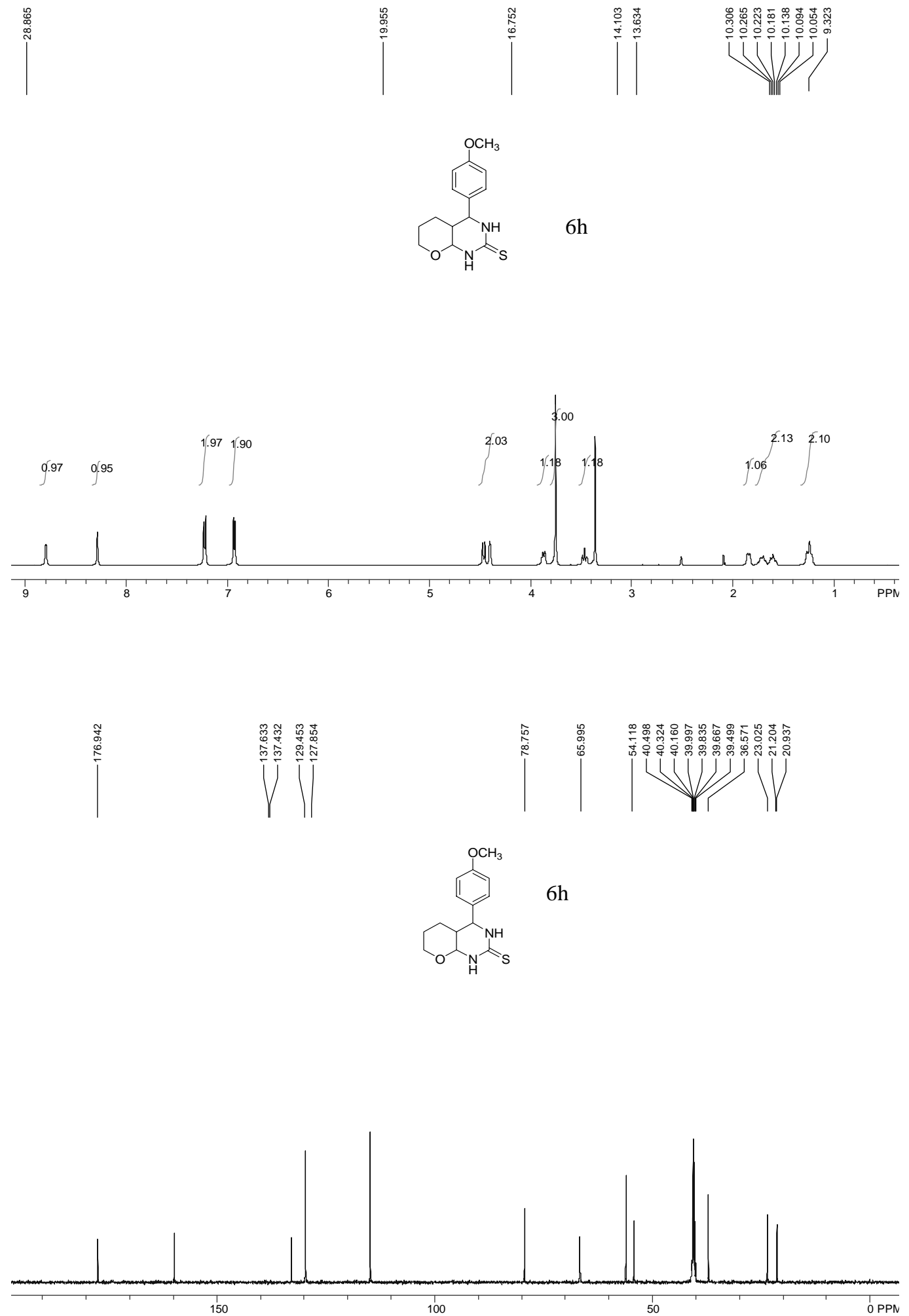

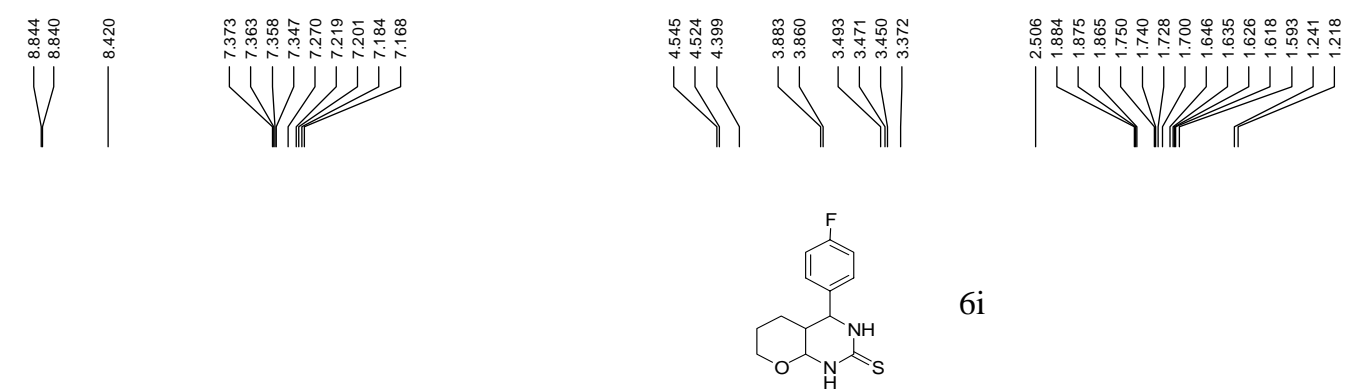

$6 \mathrm{i}$
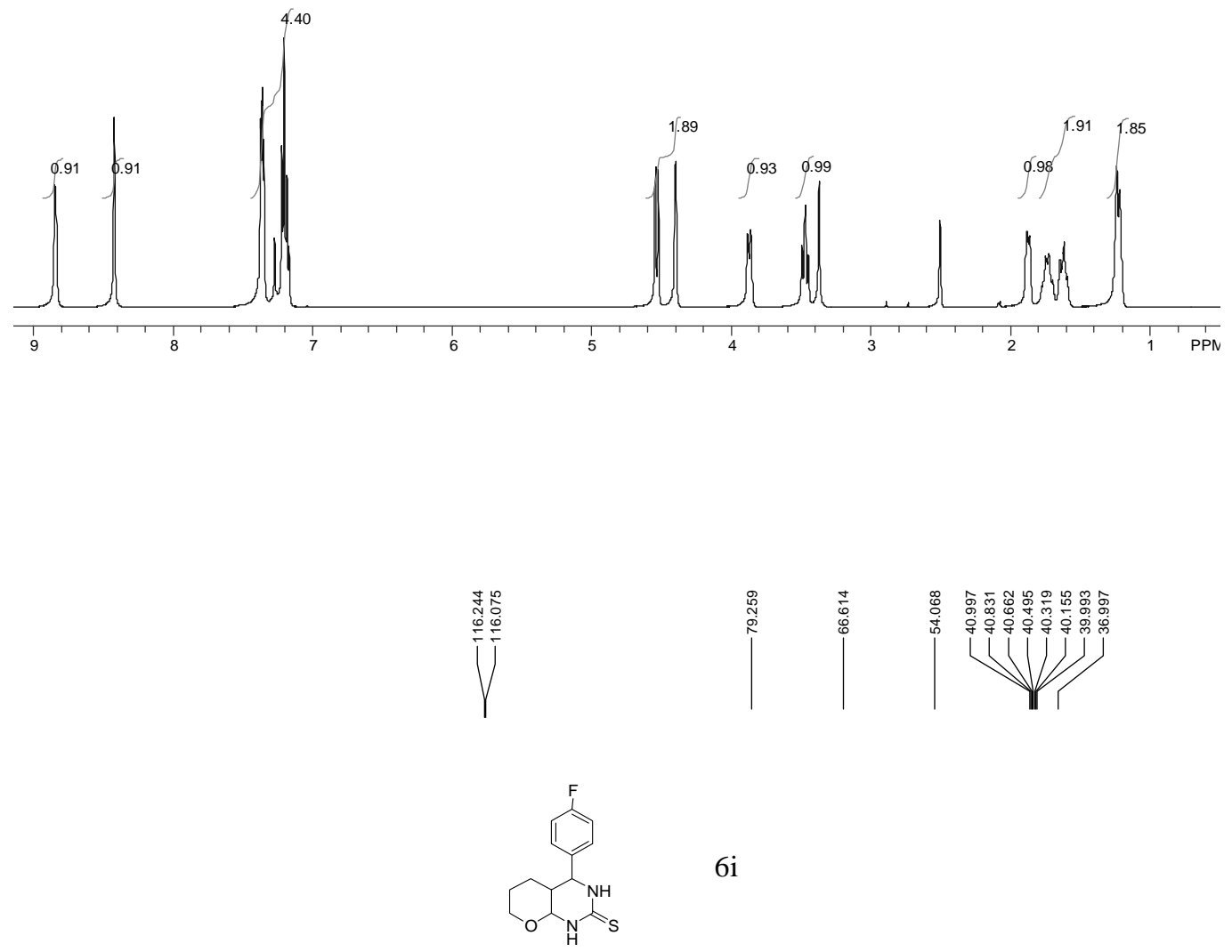

$6 i$

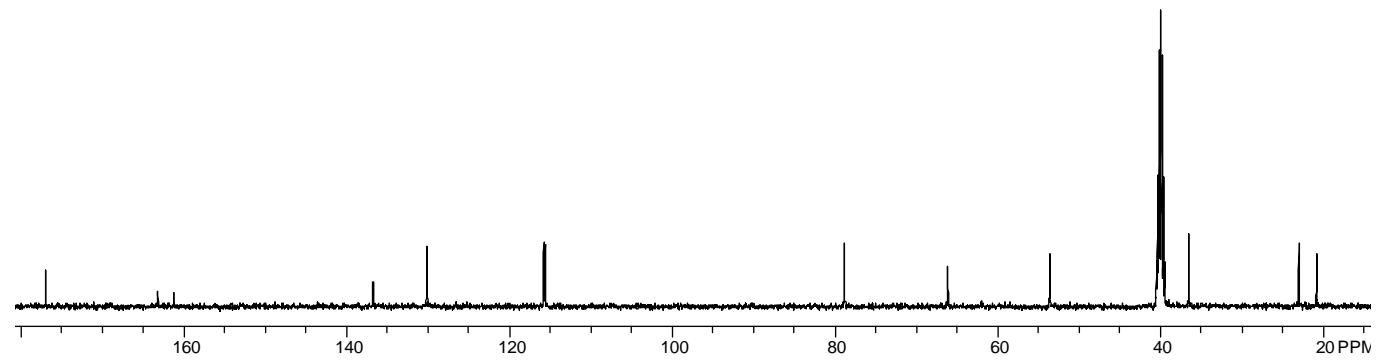



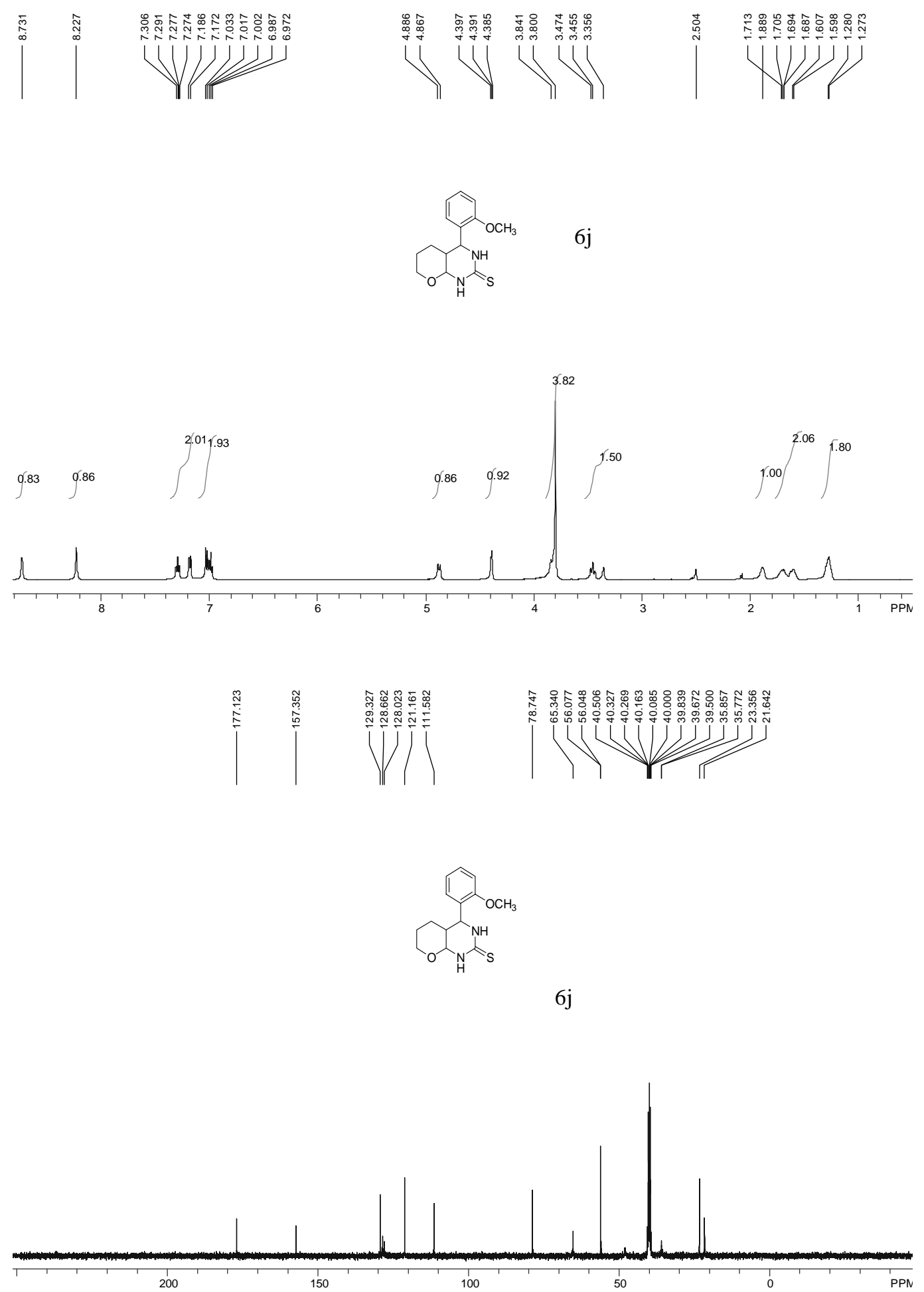

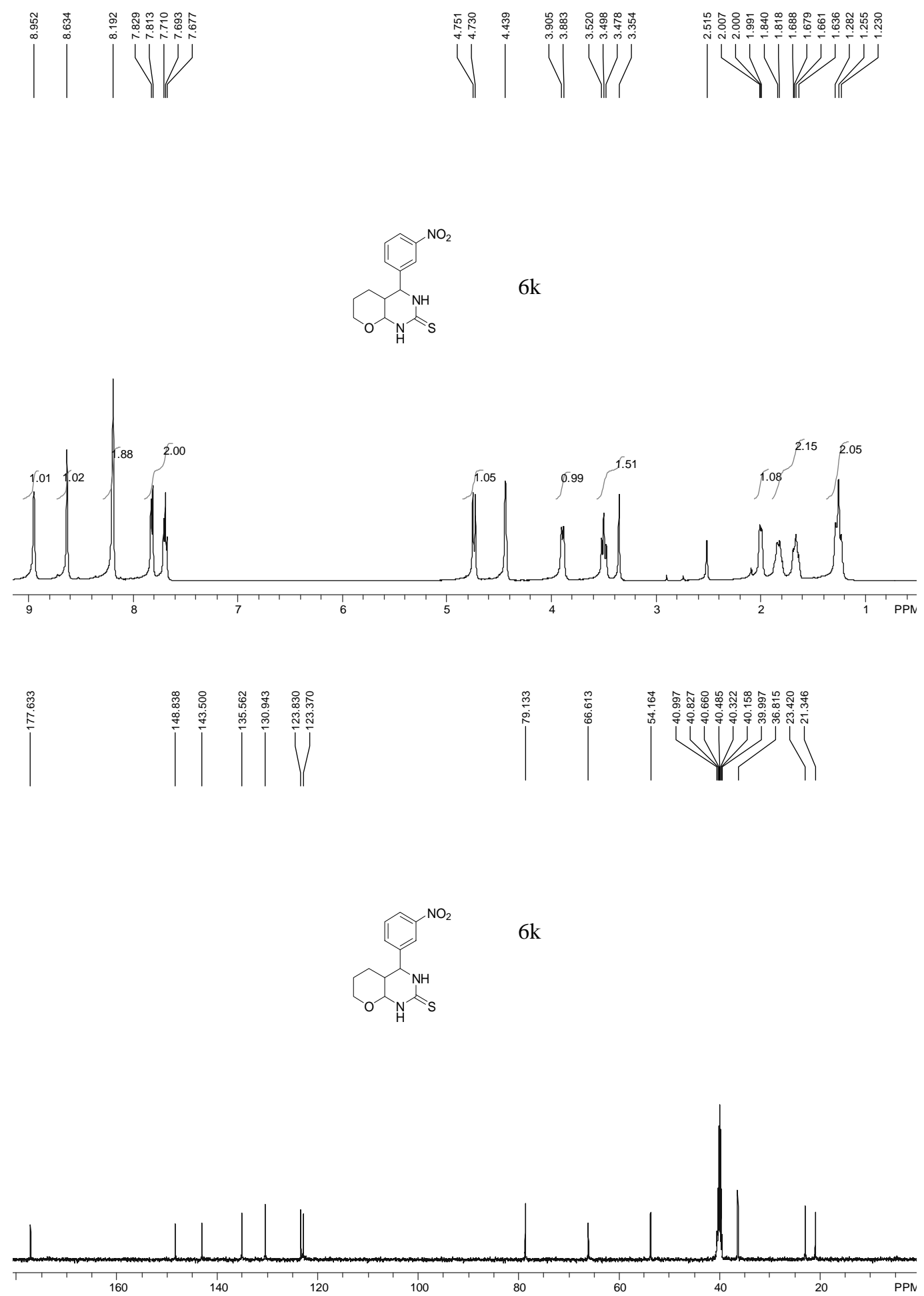